\title{
Initial conditions for hydrodynamics from weakly coupled pre-equilibrium evolution
}

\author{
Liam Keegan, ${ }^{a}$ Aleksi Kurkela, ${ }^{a, b}$ Aleksas Mazeliauskas ${ }^{c}$ and Derek Teaney ${ }^{c}$ \\ a Theoretical Physics Department, CERN, \\ CH-1211 Geneva, Switzerland \\ ${ }^{b}$ Faculty of Science and Technology, University of Stavanger, \\ 4036 Stavanger, Norway \\ ${ }^{c}$ Department of Physics and Astronomy, Stony Brook University, \\ Stony Brook, New York 11794, U.S.A. \\ E-mail: liam.keegan@cern.ch, a.k@cern.ch, \\ aleksas.mazeliauskas@stonybrook.edu, derek.teaney@stonybrook.edu
}

ABSTRACT: We use effective kinetic theory, accurate at weak coupling, to simulate the preequilibrium evolution of transverse energy and flow perturbations in heavy-ion collisions. We provide a Green function which propagates the initial perturbations to the energymomentum tensor at a time when hydrodynamics becomes applicable. With this map, the complete pre-thermal evolution from saturated nuclei to hydrodynamics can be modelled in a perturbatively controlled way.

KEYwords: Quark-Gluon Plasma, Thermal Field Theory

ARXiv EPRINT: 1605.04287 


\section{Contents}

1 Introduction 1

2 Linearized kinetic theory $\quad 4$

2.1 Setup 4

2.2 Hydrodynamization close to equilibrium 5

3 Hydrodynamization of fluctuations far from equilibrium $\quad 6$

$\begin{array}{ll}3.1 & \text { Evolution of the background energy density } \\ 3.2 & 8\end{array}$

$\begin{array}{ll}3.2 \text { Evolution of the perturbations } & 10\end{array}$

4 A Green function for hydrodynamics $\quad 12$

$\begin{array}{lll}4.1 & \text { The kinetic theory response at asymptotically small } k & 12\end{array}$

$\begin{array}{lll}4.2 & \text { Response in coordinate space } & 14\end{array}$

$\begin{array}{lll}5 & \text { Discussion } & 16\end{array}$

$\begin{array}{lr}\text { A Collision kernel } & 18\end{array}$

B Fourier transform of Green functions $\quad 20$

$\begin{array}{ll}\text { C Initial velocity perturbations } & 22\end{array}$

\section{Introduction}

Viscous relativistic hydrodynamics provides a remarkably detailed and phenomenologically successful description of the expansion of the Quark Gluon Plasma (QGP) in the ultrarelativistic heavy-ion collisions realized at the BNL Relativistic Heavy Ion Collider (RHIC) and at the CERN Large Hadron Collider (LHC) [1-3]. Hydrodynamics is an effective theory based on an assumption that the medium is sufficiently close to local thermal equilibrium that the full stress tensor can be expanded in gradients of the energy and momentum densities [4]. However, due to the singular geometry of heavy ion collisions, the gradients diverge at early times, and the hydrodynamic approach does not apply during the initial stages of the evolution. Indeed, hydrodynamic simulations start at some sufficiently late initialization time $\tau_{\text {init }} \sim 1 \mathrm{fm} / c$, when the gradient expansion becomes a useful approximation scheme. The initial conditions for hydrodynamics at $\tau_{\text {init }}$ are generally unknown, and must be parametrized and fitted to data [5]. This procedure often neglects any prethermal evolution, and limits the empirical determination of the transport coefficients of the QGP [6].

A useful prethermal model should smoothly and automatically approach hydrodynamics. If this is the case, the combined pre-thermal and hydrodynamic evolutions will be 
independent of the initialization time [7-9]. In most simulations the prethermal evolution is either completely neglected [10], or modelled in a way that does not contain the correct physics to produce hydrodynamic flow $[6,11,12]$. In addition, in some models (such as the successful IP-glasma model [12] motivated by parton saturation) the initial conditions contain strong gradients which limit the effectiveness of the hydrodynamic derivative expansion $[13,14]$. Different hydrodynamic codes regulate these extreme initial conditions in different ad hoc ways, e.g. by arbitrarily setting the shear stress tensor to zero when the hydrodynamics is initialized. Again, these ambiguities limit the ability of hydrodynamic simulations to determine the transport properties of the QGP.

In the limit of weak coupling $\alpha_{s} \ll 1$ the approach to hydrodynamics, or hydrodynamization, is described by an effective kinetic theory (EKT) [15], which takes into account the non-trivial in-medium dynamics of screening and the Landau-Pomeranchuk-Migdal suppression of collinear radiation. In ref. [16] (which includes one of the authors), it was shown that the EKT, starting with initial conditions motivated by the Color-Glass Condensate (CGC) saturation framework [17-21], reaches hydrodynamics in a phenomenologically reasonable time scale of $\sim 10 / Q_{s}$, where $Q_{s}$ is the (adjoint representation) saturation scale, which is estimated to be of order of few $\mathrm{GeV}$ for central heavy-ion collisions at the LHC. This first calculation used the EKT to monitor the equilibration of a uniform plasma of infinite transverse extent during a Bjorken expansion.

Transverse gradients in the profile will initiate flow during the equilibration process. This preflow and the accompanying modifications of the initial energy density profile will influence the subsequent hydrodynamic evolution. The goal of the current paper is to use the EKT to precisely determine the preflow and the components of the energy momentum tensor $T_{\mu}{ }^{\nu}\left(\tau_{\text {init }}, \mathbf{x}_{\perp}\right)$ that should be used to initiate the hydrodynamic evolution for a given initial energy density profile. Although the kinetic theory calculation can be used to match different models for the initial energy profile to hydrodynamics, the weak coupling approximations made in the IP-glasma model lead naturally to effective kinetic theory.

Figure 1 shows a typical transverse (entropy) profile that is used in current hydrodynamic simulations [22]. Clearly during the equilibration process the profile will change and generate initial flow. The equilibration time, $c \tau_{\text {init }}$, is short compared to the nuclear radius, $R$. For this reason the prethermal evolution is insensitive to the global collision geometry. Indeed, we may decompose the transverse plane into causally disconnected patches of size $c \tau_{\text {init }} \ll R$ whose prethermal evolution can be separately determined. In these patches, the global nuclear geometry determines a small gradient that can be considered as a linear perturbation over a translationally invariant background. Thus, corrections to initial conditions for hydrodynamics from the global geometry are of order $c \tau_{\text {init }} / R$ [23]. In addition to the global geometry, the initial energy density profile includes event-byevent fluctuations at smaller scales set by the nucleon size $R_{p}$, which is comparable to the causal horizon $R_{p} \sim c \tau_{\text {init }}$. Event-by-event fluctuations at these length scales are suppressed by $1 / \sqrt{N_{\text {part }}}$ where $N_{\text {part }}$ is the number of participating nucleons in the event, $N_{\text {part }} \sim 100-300$. Therefore, such fluctuations can also be treated in a linearized way as fluctuations over a translationally invariant background. The structure of the initial profile at even smaller scales is less well known, but in models based on CGC, one expects fluctuations to subnuclear scales of order the saturation momentum, $Q_{s}^{-1} \sim 0.1 \mathrm{fm}$. 


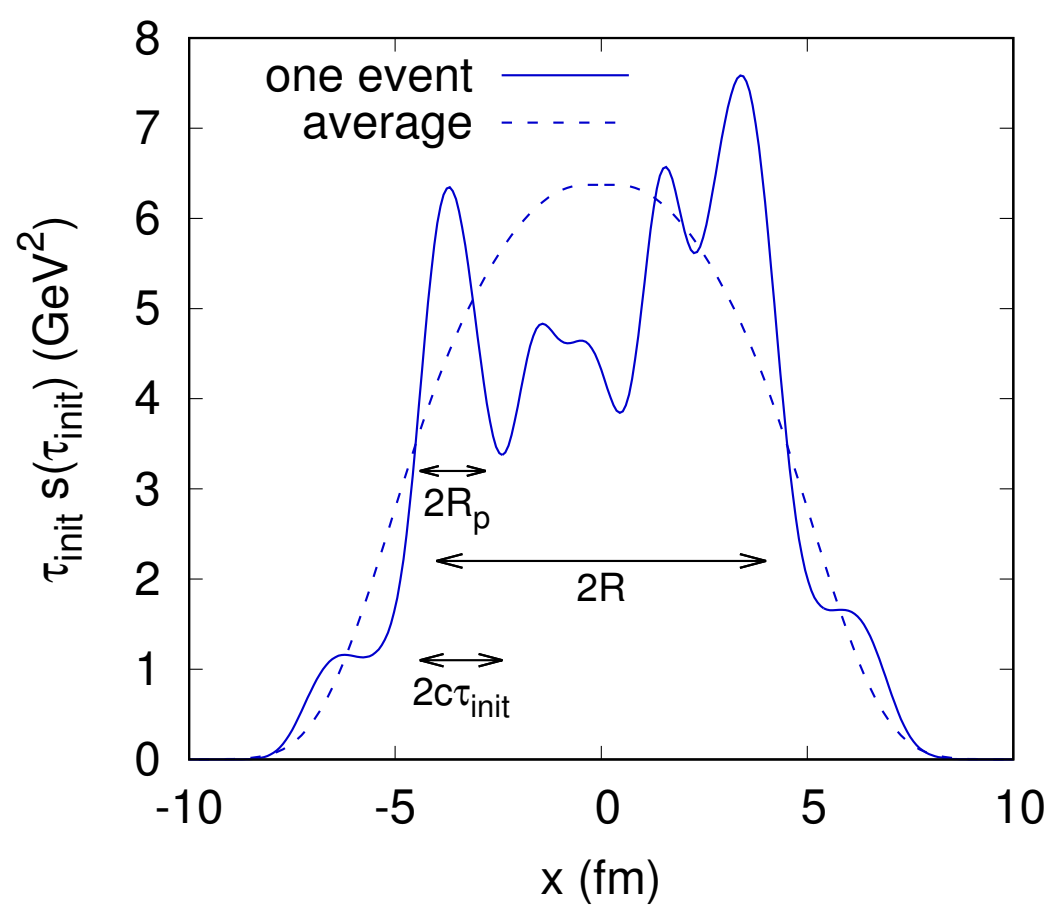

Figure 1. A typical entropy density profile (times $\tau_{\text {init }}$ ) for a single event used as an initial condition in current hydrodynamic simulations at the LHC for a $0-5 \%$ centrality class [22]. An event averaged initial condition is shown by the dashed line. Often the initial flow velocity is set to zero. The different scales are discussed in the text.

Finally, an important scale is set by the mean free path, which in a weakly coupled theory is of order $1 / \lambda^{2} T_{\text {eff }}$ for states not too far from equilibrium. In practice, this length scale is comparable, though slightly shorter than the causal horizon and the nucleon scales. Without the scale separation, the medium prethermal response to initial perturbations in the transverse plane can only be computed by a calculation within the EKT. Fortunately, as discussed above linearized kinetic theory is sufficient to determine this response.

To summarize, our strategy is to use linearized kinetic theory to follow the hydrodynamization of perturbations on top of a far-from-equilibrium Bjorken background with translational symmetry in the transverse directions. This determines the stress tensor for hydrodynamics at the initialization time. The length scales of relevance are the nucleargeometry, the nucleonic scale, the causal horizon $c \tau_{\text {init }}$, and the mean free path

$$
R \gg R_{p} \sim c \tau_{\text {init }} \sim \frac{1}{\lambda^{2} T_{\text {eff }}} .
$$

By linearizing the problem and solving for the response, we will determine a Green function describing how an energy fluctuation at the earliest moments, $\tau \sim 1 / Q_{s}$, evolves during the equilibration process to the hydrodynamic fields, i.e. the energy and momentum densities, $\delta T^{00}\left(\tau_{\text {init }}, \mathbf{x}_{\perp}\right)$ and $\delta T^{0 i}\left(\tau_{\text {init }}, \mathbf{x}_{\perp}\right)$ respectively. We will verify that the subsequent evolution is described by second order hydrodynamics to certifiable precision.

In section 2 we outline the linearized EKT, and study the linear response of the EKT in equilibrium. In section 3 we systematically study the approach to equilibrium of Fourier 
modes of specified $k$, starting with a far from equilibrium initial state. In section 4 we Fourier transform these results and determine a coordinate space Green function which produces the appropriate initial conditions for hydrodynamics at $\tau_{\text {init }}$ when convolved with a specified initial state. We also analyze the long wavelength limit of these Green functions, making contact and providing additional insight into previous work on preflow [23]. Finally, we discuss our conclusions in section 5 .

\section{Linearized kinetic theory}

\subsection{Setup}

At weak coupling the non-equilibrium evolution of the boost invariant color and spin averaged gluon distribution function is described in terms of an effective kinetic equation [15]

$$
\partial_{\tau} f_{\mathbf{x}_{\perp}, \mathbf{p}}+\frac{\mathbf{p}}{|p|} \cdot \nabla_{\mathbf{x}_{\perp}} f_{\mathbf{x}_{\perp}, \mathbf{p}}-\frac{p_{z}}{\tau} \partial_{p_{z}} f_{\mathbf{x}_{\perp}, \mathbf{p}}=-\mathcal{C}\left[f_{\mathbf{x}_{\perp}, \mathbf{p}}\right],
$$

where the effective collision kernel $\mathcal{C}[f]$ incorporates the elastic $2 \leftrightarrow 2$ and inelastic $1 \leftrightarrow 2$ processes as required for a leading order description in the coupling constant $\lambda=4 \pi \alpha_{s} N_{c}$, which is the only parameter of the EKT. The kinetic theory is valid when the occupancies are perturbative $\lambda f \ll 1$ and when the relevant distance scales are larger than the typical Compton wavelength of the particles $\Delta x \gtrsim\langle p\rangle^{-1}$. The details of the scattering kernel have been discussed in refs. $[15,16,24]$ and are briefly repeated here in the appendix A. We use the isotropic screening approximation from [16] which is leading order accurate for parametrically isotropic systems $\mathcal{P}_{L} / \mathcal{P}_{T} \approx 1$. (Here and below $\mathcal{P}_{L}$ and $\mathcal{P}_{T}$ denote the longitudinal and transverse pressures.) In the current paper we will consider only gluonic degrees of freedom and assume that the contribution of quarks is suppressed during the pre-equilibrium evolution. ${ }^{1}$

We split the distribution function into a translationally symmetric background and a linearized perturbation with a wavenumber $\mathbf{k}_{\perp}$ in the transverse plane

$$
f_{\mathbf{x}_{\perp}, \mathbf{p}}=\bar{f}_{\mathbf{p}}+\int \frac{d^{2} \mathbf{k}_{\perp}}{(2 \pi)^{2}} A\left(\mathbf{k}_{\perp}\right) e^{i \mathbf{k}_{\perp} \cdot \mathbf{x}_{\perp}} \delta f_{\mathbf{k}_{\perp}, \mathbf{p}}
$$

where $A\left(\mathbf{k}_{\perp}\right)$ characterizes the initial density profile. The kinetic equations for the background and the (complex) fluctuation then read

$$
\begin{aligned}
\left(\partial_{\tau}-\frac{p_{z}}{\tau} \partial_{p_{z}}\right) \bar{f}_{\mathbf{p}} & =-\mathcal{C}[\bar{f}] \\
\left(\partial_{\tau}-\frac{p_{z}}{\tau} \partial_{p_{z}}+\frac{i \mathbf{p}_{\perp} \cdot \mathbf{k}_{\perp}}{p}\right) \delta f_{\mathbf{k}_{\perp}, \mathbf{p}} & =-\mathcal{C}[\bar{f}, \delta f]
\end{aligned}
$$

where $\mathcal{C}[\bar{f}, \delta f]$ is the collision kernel linearized in $\delta f$ (see appendix A for details).

\footnotetext{
${ }^{1}$ The initial far-from-equilibrium state is parametrically dominated by gluons. Once the plasma has thermalized it should contain also fermionic degrees of freedom. However, the production of fermions is suppressed by larger color factors $C_{F} / C_{A}$, and by Pauli blocking factors (while scattering of gluons is Bose enchanced). It is therefore plausible that the system hydrodynamizes before it is chemically equilibrated.
} 


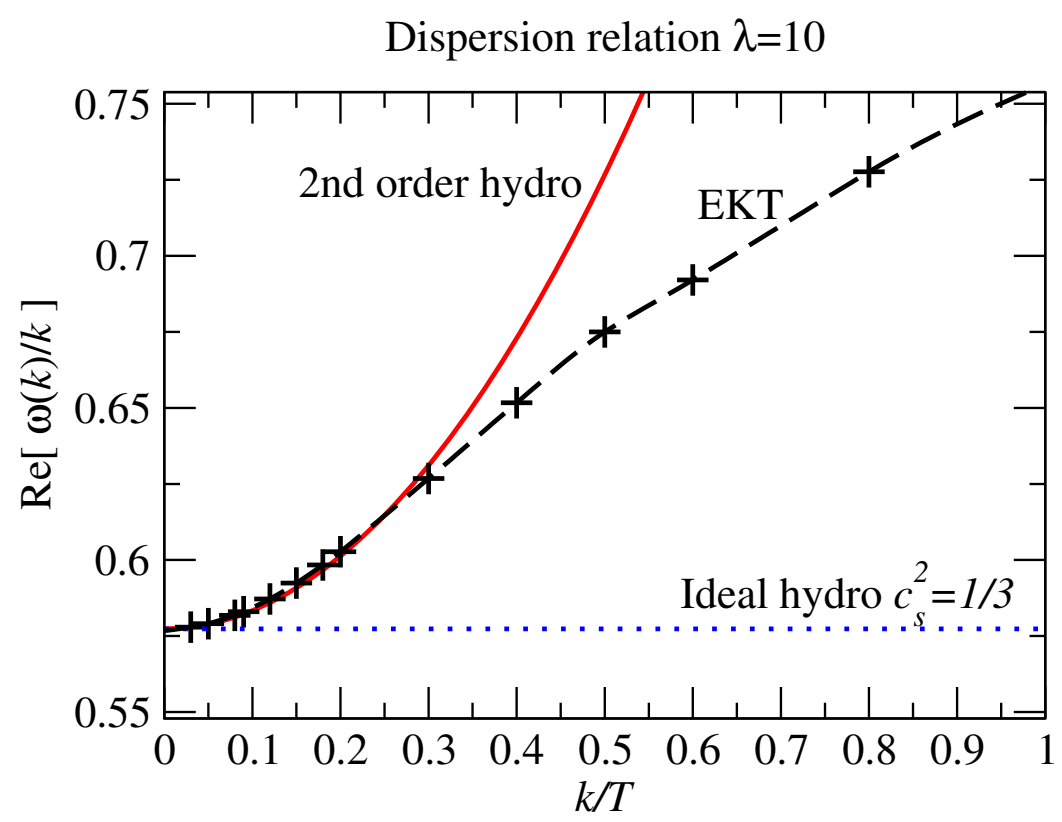

Figure 2. The dispersion relation of sound modes with thermal background from the EKT. The long wavelength modes are described by ideal hydrodynamics with $\omega=c_{s} k$ and $c_{s}^{2}=1 / 3$, and the approach to ideal hydrodynamics is well described by 2 nd order hydrodynamics. For modes with wave numbers larger than $k \gtrsim 0.4 T$, the dispersion relation differs significantly from the hydrodynamic expectation.

\subsection{Hydrodynamization close to equilibrium}

Before studying the equilibration process, we will analyze the linear response of the EKT close to equilibrium, corresponding to the $\tau \rightarrow \infty$ limit of (2.3). Our goal in this section is to determine at what wavenumbers (characterized by $k / T$ ) linearized energy-momentum perturbations are described by hydrodynamics for an equilibrated background.

The dispersion relation for the sound mode to second order in the hydrodynamic expansion reads [4]

$$
\omega=c_{s} k-i \frac{4}{3} \frac{\eta}{e+p} k^{2}+\frac{4}{3} \frac{\eta}{e+p}\left(c_{s} \tau_{\pi}-\frac{2}{3 c_{s}} \frac{\eta}{e+p}\right) k^{3},
$$

where $c_{s}^{2}=1 / 3$ for conformal equation of state and $\eta, \tau_{\pi}$, are known transport coefficients at weak coupling [25, 26]. For $\lambda=10$ (corresponding to $\alpha_{s} \approx 0.26$ ) the hydrodynamic coefficients read $\eta / s=0.62, \tau_{\pi}=5.1 \eta / s T$, and $\lambda_{1}=0.8 \eta \tau_{\pi}$. We will quantify at what numerical values of $k / T$ the corrections to (2.4) become sizeable.

To this end, the kinetic theory is initiated in local thermal equilibrium with a spatially varying temperature, $T(\mathbf{x})=T+\delta T e^{i \mathbf{k}_{\perp} \cdot \mathbf{x}_{\perp}}$, and corresponding phase space distribution

$$
\delta f_{\mathbf{k}_{\perp}, \mathbf{p}}^{(1)}=-\frac{\delta T}{T} p \partial_{p} \bar{f}_{\mathbf{p}}, \quad \text { and } \quad \bar{f}_{\mathbf{p}}=\frac{1}{e^{p / T}-1} .
$$

Gradients in the energy density drive momentum perturbations in due course, and the frequency of the subsequent (damped) oscillations determines the real part of the dispersion 
relation. Numerically, we obtained the oscillation frequency by measuring the time interval between the successive nodes.

The results for various $k / T$ are depicted in figure 2 for $\lambda=10$. We see that at small $k$, $k / T \lesssim 0.1$, the dispersion relation is well described by the $c_{s}^{2}=1 / 3$ result of ideal hydrodynamics, and the approach to ideal hydrodynamics is described by the 2nd order corrections of (2.4) (note that the real part of the frequency does not get a first order correction). Indeed, for $k / T \lesssim 0.4$, the second order hydrodynamic theory matches well with the EKT.

At higher values of $k / T$, the EKT finally saturates at $\omega=k$ in contrast to the strict (unresummed) second order hydrodynamics. For $\lambda=10$ this happens only at rather large values of $k, k>T$. For these wavenumbers, the wavelength of the perturbation is comparable to the typical gluon Compton wavelength, and the linear response of the system cannot be reliably computed with kinetic theory in this regime.

We conclude that for $\lambda=10$, the smallest scales that hydrodynamize have $k \sim 0.4 T$. Varying the value of $\lambda<10$ (not shown), we find that the scale where hydrodynamics breaks downs tracks the shear viscosity, $k \sim 0.4[\eta(\lambda=10) / \eta(\lambda)] T$ with varying $\lambda$. Note that for smaller $\lambda$, the saturation to $\omega \sim k$ can take place within the regime of validity of the effective theory.

\section{Hydrodynamization of fluctuations far from equilibrium}

We now move on to study the hydrodynamization of spatially dependent fluctuations on top of a far-from-equilibrium boost invariant background. As discussed in [16, 27-29], at very early times $\tau \lesssim Q_{s}^{-1}$ the far-from-equilibrium gluonic system in the midrapidity region is parametrically over-occupied $\lambda f \sim 1$, and the dynamics is described with coherent classical gauge fields rather than with particles. This part of the evolution is characterized by negative values of the longitudinal pressure $\mathcal{P}_{L}$, which is a result of the coherence of the approximately boost invariant fields. However, classical numerical simulations [21, 29] (as well as analytical series solutions to the classical equations of motion [30, 31]) show that in a timescale $Q_{s} \tau \sim 1$, the coherence is lost, the longitudinal pressure approaches zero $\mathcal{P}_{L} \sim 0$, and the occupancies become perturbative [32-35]. At this point the system may be passed to the EKT [16, 36-38].

Following [16], we take as our initial condition at $\tau_{0}=1 / Q_{s}$ a parametrization

$$
\begin{aligned}
f\left(p_{z}, p_{\perp}\right) & =\frac{2}{\lambda} A f_{0}\left(p_{z} \xi / p_{0}, p_{\perp} / p_{0}\right), \\
f_{0}\left(\hat{p}_{z}, \hat{p}_{\perp}\right) & =\frac{1}{\sqrt{\hat{p}_{\perp}^{2}+\hat{p}_{z}^{2}}} e^{-2\left(\hat{p}_{\perp}^{2}+\hat{p}_{z}^{2}\right) / 3}
\end{aligned}
$$

where $p_{0}=1.8 Q_{s}, \xi=10$, and $\lambda=10$. The parameters $p_{0}$ and $\xi$ are motivated by classical simulations where $\sqrt{\left\langle p_{T}^{2}\right\rangle} \approx 1.8 Q_{s}$ and $\left\langle p_{z}^{2}\right\rangle \ll\left\langle p_{T}^{2}\right\rangle$. The amplitude, $A$, is adjusted so that energy per rapidity

$$
\tau_{0} e\left(\tau_{0}\right)=\tau_{0} \nu_{g} \int \frac{d^{3} p}{(2 \pi)^{3}}|p| f\left(p_{z}, p_{\perp}\right)
$$


matches the results of classical simulations [21], where

$$
\tau_{0} e\left(\tau_{0}\right) \simeq 0.358 \frac{\tau_{0} \nu_{g} Q_{s}^{4}}{\lambda}
$$

Here $\nu_{g}=2 d_{A}=16$ is the number of gluonic degrees of freedom. With these parameters, the number of gluons and the mean $p_{T}$ in the EKT at $\tau_{0}$ are

$$
\frac{d N}{d^{2} \mathbf{x}_{\perp} d y}=0.232 \frac{\nu_{g} Q_{s}^{2}}{\lambda}, \quad \sqrt{\left\langle p_{T}^{2}\right\rangle}=1.8 Q_{s},
$$

which roughly matches the classical Yang-Mills simulations.

We will follow the response to two specific initial perturbations of these initial conditions, which provide an independent basis for describing energy and momentum fluctuations in the transverse plane. For the energy fluctuation, we take

$$
\delta f_{\mathbf{k}_{\perp}, \mathbf{p}}^{(1)}\left(\tau_{0}\right)=-\frac{\delta Q_{s}}{Q_{s}} p \partial_{p} \bar{f}_{\mathbf{p}}
$$

which results from varying the saturation scale in $(3.1), Q_{s}\left(\mathbf{x}_{\perp}\right) \sim Q_{s}+\delta Q_{s} e^{i \mathbf{k}_{\perp} \cdot \mathbf{x}_{\perp}}$. This initial condition is studied in the bulk of the paper. For the momentum fluctuation we take

$$
\delta f_{\mathbf{k}_{\perp}, \mathbf{p}}^{(2)}\left(\tau_{0}\right)=-\frac{\delta Q_{s}}{Q_{s}} \hat{\mathbf{k}}_{\perp} \cdot \partial_{\mathbf{p}} \bar{f}_{\mathbf{p}}
$$

which does not perturb the local energy density. The response to this initial condition is recorded in appendix $\mathrm{C}$.

Without loss of generality, we can choose the wave vector $\hat{k}_{\perp}=(k, 0)$ to point in $x$-direction. Then at any time, the energy and momentum perturbations are defined as

$$
\begin{aligned}
& \delta e(\tau, k) \equiv \delta T^{00}=\nu_{g} \int \frac{d^{3} \mathbf{p}}{(2 \pi)^{3}} p^{0} \delta f \\
& g^{x}(\tau, k) \equiv \delta T^{0 x}=\nu_{g} \int \frac{d^{3} \mathbf{p}}{(2 \pi)^{3}} p^{x} \delta f
\end{aligned}
$$

and their evolution is governed by the linearized conservation equations

$$
\begin{aligned}
\partial_{\tau} e(\tau) & =-\frac{e(\tau)+T^{z z}(\tau)}{\tau}, \\
\partial_{\tau} \delta e(\tau, k)+i k g^{x}(\tau, k) & =-\frac{\delta e(\tau, k)+\delta T^{z z}(\tau, k)}{\tau}, \\
\partial_{\tau} g^{x}(\tau, k)+i k \delta T^{x x}(\tau, k) & =-\frac{g^{x}(\tau, k)}{\tau},
\end{aligned}
$$

where $\delta T^{\mu \nu}$ is the energy-momentum tensor perturbation caused by $\delta f$. If the system is described by hydrodynamics then $T^{z z}, \delta T^{z z}$ and $\delta T^{x x}$ are determined through the constitutive equations by the first moments of the particle distribution function $e, \delta e$ and $g^{x}$. 

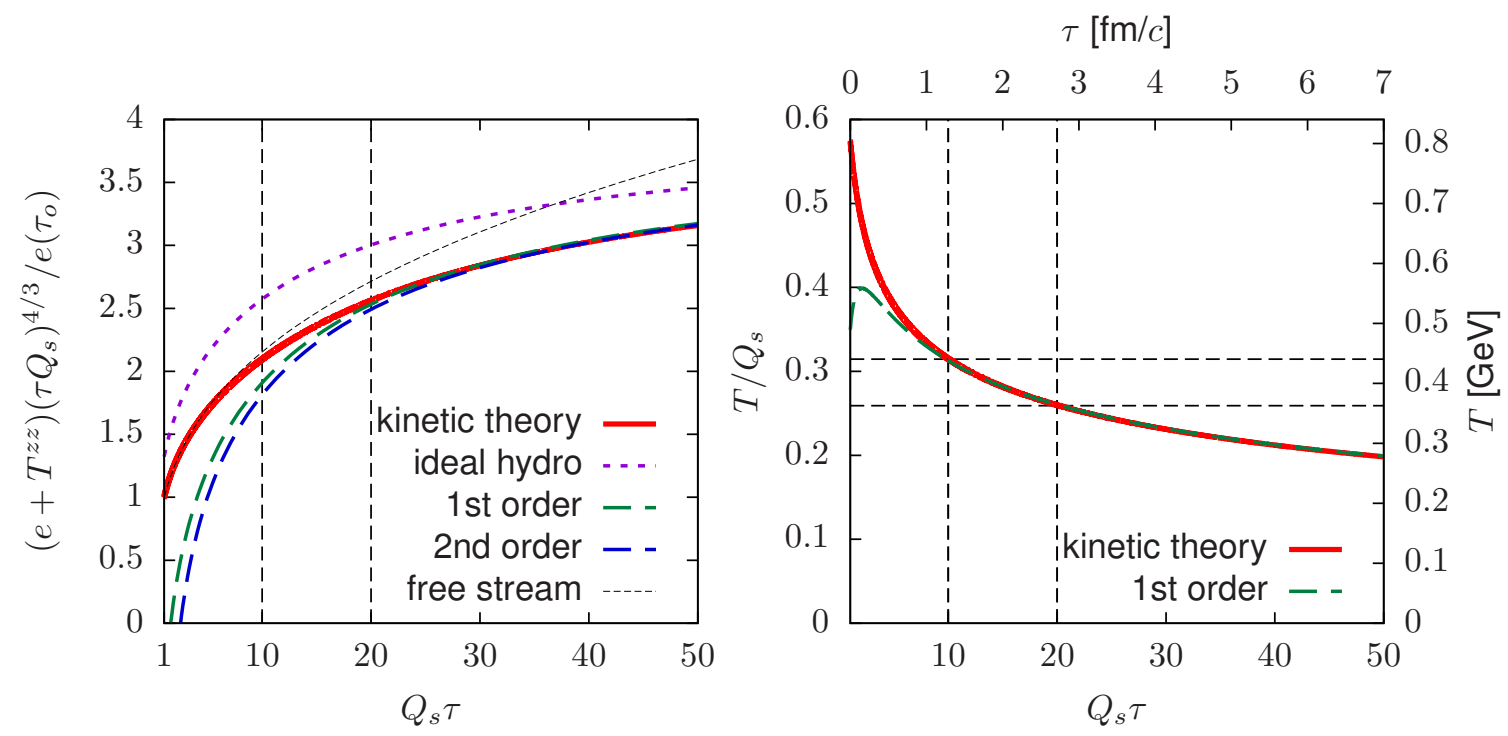

Figure 3. (a) A comparison of the relevant combination $e(\tau)+T^{z z}(\tau)$ for the kinetic theory background with the hydrodynamic constitutive equations of (3.11a). (b) The background effective temperature as obtained from the Landau matching condition $e=\nu_{g} \frac{\pi^{2}}{30} T^{4}$. Extrapolation of first order hydro (fitted at asymptotic late times) is shown for comparison, (3.16). The scales in physical units correspond to $Q_{s}=1.4 \mathrm{GeV}$ which yields the entropy required by hydrodynamic simulations (see text).

For conformal second order viscous hydrodynamics these relations are

$$
\begin{aligned}
T^{z z}(\tau)= & \frac{1}{3} e-\frac{4}{3} \frac{\eta}{\tau}-\frac{8}{9} \frac{\tau_{\pi} \eta-\lambda_{1}}{\tau^{2}}, \\
\delta T^{x x}(\tau, k)= & \frac{\delta e(\tau, k)}{e}\left[\frac{1}{3} e+\frac{1}{3} \eta \tau_{\pi} k^{2}+\frac{1}{2 \tau} \eta-\frac{2\left(\lambda_{1}-\eta \tau_{\pi}\right)}{9 \tau^{2}}\right] \\
& -i \frac{k g^{x}(\tau, k)}{e}\left[\eta-\frac{1}{\tau}\left(\frac{\eta^{2}}{2 e}+\frac{\eta \tau_{\pi}}{2}-\frac{2}{3} \lambda_{1}\right)\right] \\
\delta T^{z z}(\tau, k)= & \frac{\delta e(\tau, k)}{e}\left[\frac{1}{3} e-\frac{1}{6} \eta \tau_{\pi} k^{2}-\frac{1}{\tau} \eta+\frac{4\left(\lambda_{1}-\eta \tau_{\pi}\right)}{9 \tau^{2}}\right] \\
& +i \frac{k g^{x}(\tau, k)}{e}\left[\frac{1}{2} \eta-\frac{1}{\tau}\left(\frac{\eta^{2}}{4 e}+\frac{2}{3} \lambda_{1}\right)\right],
\end{aligned}
$$

where the constitutive equations for ideal (or first order viscous) hydrodynamics can be recovered by setting $\eta=\tau_{\pi}=\lambda_{1}=0$ (or $\tau_{\pi}=\lambda_{1}=0$ ).

\subsection{Evolution of the background energy density}

Before studying the perturbations, we will study the equilibration of the background energy density, elaborating on the original study [16]. In figure 3(a) we compare the energy momentum tensor combination $e+T^{z z}$ in the kinetic theory simulation to the constitutive equation, (3.11a). The $e+T^{z z}$ combination is motivated by the conservation law in (3.10a). 
At early times the system evolves approximately according to free streaming, ${ }^{2}$ with $T^{z z} \sim 0$ and $e \propto \tau^{-1}$. As already noticed in [16], the constitutive equations give an increasingly accurate description of the EKT stress tensor as a function of time. While the ideal constitutive equations are rather far from the EKT at all relevant times, the viscous and 2nd order equations quickly converge to the EKT. Note that an accidental (approximate) cancellation of $\lambda_{1}-\eta \tau_{\pi}$ makes the second order correction anomalously small [39], and only at rather late times after non-hydrodynamic modes have almost completely decayed does second order hydrodynamics finally improve the first order result (not shown). By times $Q_{s} \tau=\{10,20\}$, the viscous constitutive equations agree with the EKT within $\{10 \%, 2 \%\}$.

It is noteworthy that the EKT interpolates smoothly between the free streaming and viscous hydrodynamic evolutions without an extended period during which the evolution is not approximately described by one or the other approximation scheme. At $Q_{s} \tau=10$ the evolution is somewhat closer to free streaming, and using hydrodynamics at this point is a rough, though perhaps acceptable, approximation. At $Q_{s} \tau=20$ hydrodynamics is a better approximation, but at this time the causal horizon is becoming comparable to the nuclear radius.

Given the agreement with the constitutive equations, one can use the hydrodynamic equations to propagate the system forward in time. At late times ideal hydrodynamics is valid, and the entropy per area per rapidity approaches a constant

$$
\lim _{\tau \rightarrow \infty} \tau s(\tau) \equiv \frac{\nu_{g} \Lambda_{s}^{2}}{\lambda}
$$

(Here the $\Lambda_{s}$ parametrization is motivated by the scaling of the initial multiplicity with the saturation scale in (3.4).) Dimensional reasoning indicates that $\Lambda_{s}^{2}$ is proportional to $Q_{s}^{2}$. Taking the data presented in figure 3(a) we may extrapolate $\tau \rightarrow \infty$ to determine the proportionality coefficient

$$
\Lambda_{s}^{2}=1.95 Q_{s}^{2} .
$$

Here we have used the ideal equation of state to convert energy density to entropy density. Since the entropy per gluon of an ideal gluon gas is 3.6, (3.13) implies the asymptotic number of gluons per area per rapidity is more than a factor of two larger than the input number of gluons at $\tau_{0}$

$$
\left.\frac{d N}{d^{2} \mathbf{x}_{\perp} d \eta}\right|_{\tau \rightarrow \infty}=\left.2.33 \frac{d N}{d^{2} \mathbf{x}_{\perp} d \eta}\right|_{\tau=\tau_{0}} .
$$

At $Q_{s} \tau=\{10,20\}$ the entropy and gluon multiplicity have reached only $\{72,82\} \%$ of their asymptotic values, corresponding to gluon multiplication factors of $\{1.6,1.9\}$ respectively.

Finally, let us make phenomenological contact with more complete hydrodynamic simulations of heavy ion collisions, and estimate the saturation momentum required by phenomenology. The initial entropy in hydrodynamics is normally adjusted to reproduce the mean multiplicity. Using the computer code from one such hydrodynamic simulation at the

\footnotetext{
${ }^{2}$ During this part of the evolution, the system evolves according to the nonthermal attractors discussed in e.g. [16, 33-35]. The nonthermal attractors are characterized by $T^{z z} \ll e$, and for the current discussion the fine details of the attractor are irrelevant, and the evolution resembles that of free streaming.
} 
LHC [22], we computed the average entropy per area at the hydrodynamic initialization time $^{3}$

$$
\left\langle\tau_{\text {init }} s\left(\tau_{\text {init }}\right)\right\rangle=4.13 \mathrm{GeV}^{2} .
$$

Entropy production during the subsequent hydrodynamic evolution is small, approximately $15 \%$, and therefore this constant is approximately independent of the initialization time. With (3.12) and (3.13), setting $Q_{s} \simeq 1.4 \mathrm{GeV}$ in the EKT will roughly reproduce the entropy in hydrodynamic simulations provided the system is passed to hydrodynamics at $Q_{s} \tau=10$ where the entropy in the EKT has reached $70 \%$ of its asymptotic value in (3.12).

In figure $3(\mathrm{~b})$ we present the time evolution of the effective temperature (determined from the energy density and the equation of state) in physical units for $Q_{s} \simeq 1.4 \mathrm{GeV}$. Its time dependence at late times is well described by first order viscous hydrodynamics with the asymptotic value

$$
\lim _{\tau \rightarrow \infty}\left(T+\frac{2}{3} \frac{\eta}{s \tau}\right) \tau^{1 / 3}=0.763 Q_{s}^{2 / 3}
$$

The temperature at $Q_{s} \tau=10$ is $T \simeq 430 \mathrm{MeV}$, or close to three times the pseudo-critical temperature. At these temperatures, modern weakly coupled techniques can be expected to work reasonably, justifying our approximation scheme. Similarly, with this value of $Q_{s}$ an initialization time of $Q_{s} \tau \simeq 10$ corresponds to $\tau_{\text {init }} \simeq 1.4 \mathrm{fm}$. The elliptic and triangular flows in central events develop on a later time scale, of order $R / c_{s} \sim 8 \mathrm{fm}$, and therefore an initialization time of this order may be acceptable. A more complete study including fermions will be needed to definitively answer this question.

\subsection{Evolution of the perturbations}

We now move on to describe the evolution of the linearized perturbations on top of the thermalizing non-equilibrium background. We first follow the evolution of an energy density perturbation, which we start with the initial condition of (3.6) with different values of $k / Q_{s}$. In figures $4(\mathrm{a})$-(d) we show $x x$-component of perturbation energy momentum tensor $\delta T^{x x}$ compared with ideal, viscous, and second order hydrodynamic constitutive equations of (3.11b). The lines have been normalized by the background energy density $T^{00}(\tau)$, so that any observed damping is due to nontrivial dynamics associated to the spatial inhomogeneity.

We consider fixed values of $k / Q_{s}$, which do not correspond to fixed values of $k / T$, as the effective temperature is changing due to the expansion (see figure 4). For very large wavelengths with $k / Q_{s}=0.01$, we observe that while ideal constitutive equations have rather large corrections, these are well accounted for by the viscous and 2 nd order equations, roughly at the same time as the background constitutive equation is satisfied, i.e. at times after $\tau \sim 10 / Q_{s}$. Determining the temperature of the background from Landau matching condition $T^{4}=\nu_{g} \frac{\pi^{2}}{30} e$ (see figure 3 (right)), we find that at times $\tau=$

\footnotetext{
${ }^{3}$ Specifically, for the event-by-event hydro code described in ref. [22] we first created a smooth entropy density profile, $\overline{s\left(\mathbf{x}_{\perp}\right)}$, by averaging over events for a $0-5 \%$ centrality class, $b=[0,3.3] \mathrm{fm}$. This average is shown in figure 1 . We then computed a single averaged entropy density by averaging $\overline{s\left(\mathbf{x}_{\perp}\right)}$ with $\overline{s\left(\mathbf{x}_{\perp}\right)}$ as a radial weight.
} 

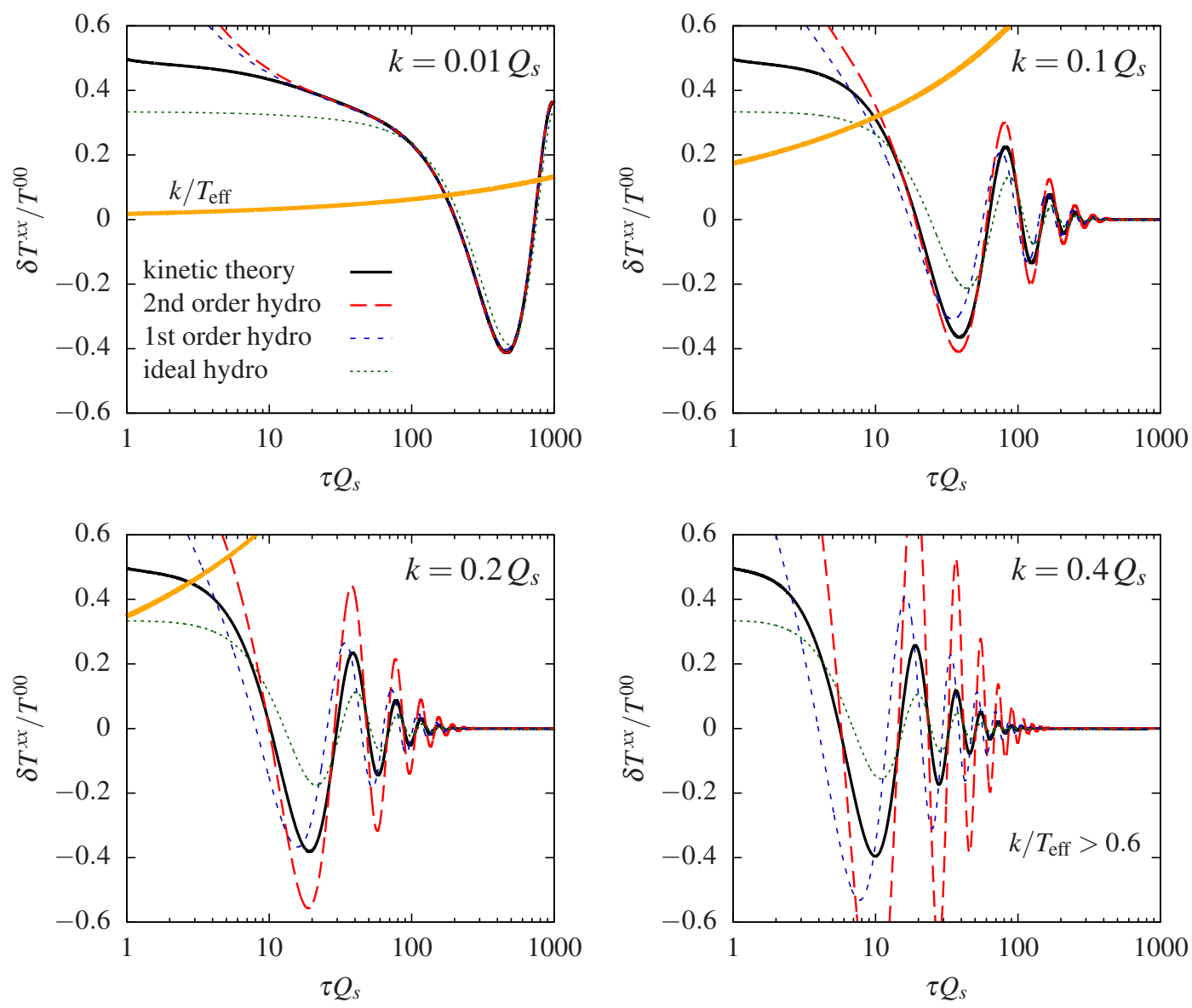

Figure 4. $\delta T^{x x} / T^{00}$ compared with hydrodynamic constitutive equations (the curves have been normalized by the magnitude of the initial perturbation $\left.\delta T^{00}\left(\tau_{0}\right) / T^{00}\left(\tau_{0}\right)\right)$. Long wavelengths with $k \lesssim 0.1 T$ are described by the hydrodynamics at approximately the same time as the background $Q_{s} \tau \sim 10$. Shorter wavelengths with $k \sim 0.4 Q_{s}$ are never well described by hydrodynamics.

$\{10,20\} / Q_{s}$, the wavelength $k=0.01 Q_{s}$ in units of temperature $T=\{0.31,0.26\} Q_{s}$ is $k / T(\tau)=\{0.032,0.039\}$. As discussed in section 2.2 and in figure 2 these values of $k / T$ are accurately described by second order hydrodynamics.

We see that even for larger $k / Q_{s}=\{0.1,0.2,0.4\}$ the hydrodynamic constitutive relations are approximately fulfilled when the background has hydrodynamized around $\tau \sim 10 / Q_{s}$. However, larger values of $k / Q$ correspond to larger values of $k / T$, and even at late times there are corrections to the constitutive equations. While these corrections are moderate for $k / Q_{s}=0.2$ for which $k / T\left(\tau=10 / Q_{s}\right) \approx 0.6$, they remain $\mathcal{O}(1)$ for $k / Q_{s}=0.4$. It is therefore questionable whether it is justifiable to pass these short scales to hydrodynamic description at any time. 
Normalized linear response at $Q_{s} \tau=10$

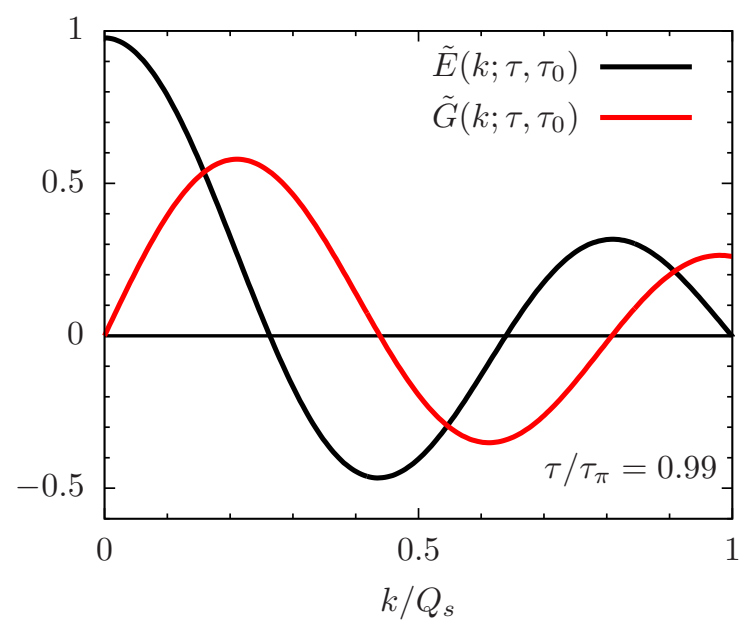

Normalized linear response at $Q_{s} \tau=20$

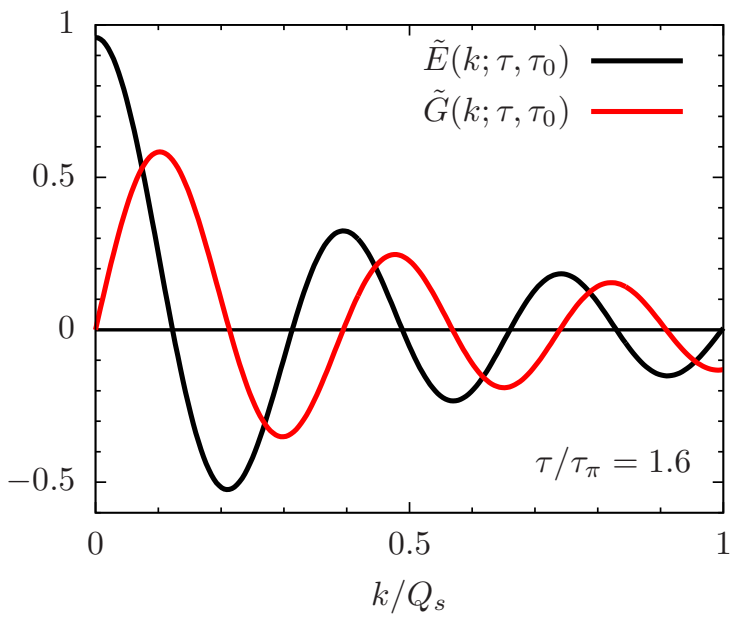

Figure 5. Normalized linear response functions in $k$-domain [(4.1) and (4.2)] for the initial energy perturbation (a) at $Q_{s} \tau=10$ and (b) at $Q_{s} \tau=20$.

\section{A Green function for hydrodynamics}

We now move on to describe how the response to the linearized perturbations in EKT can be used in a hydrodynamic simulations to encapsulate the far-from-equilibrium dynamics of transverse perturbations during the time scales between $\tau \sim 1 / Q_{s}$ and $\{10,20\} / Q_{s}$.

In order to construct the initial state for hydrodynamics at $\tau_{\text {init }}$ from a given geometry at $\tau_{0}$, the linear response of the components of $T^{\mu \nu}$ to the initial perturbation are needed. The constitutive relations reduce the number of independent components of the energy momentum tensor, so it suffices to specify only $\delta T^{00}$ and $\delta T^{0 x}$. Figure 5(a) displays the energy and momentum response functions $\left(\tilde{E}\left(k ; \tau, \tau_{0}\right)\right.$ and $\tilde{G}\left(k ; \tau, \tau_{0}\right)$ respectively) to an initial energy perturbation $\delta e\left(\tau_{0}, k\right)$ in $\mathbf{k}$-space

$$
\begin{aligned}
& \frac{\delta e(\tau, k)}{e(\tau)} \equiv \tilde{E}\left(k ; \tau, \tau_{0}\right) \frac{\delta e\left(\tau_{0}, k\right)}{e\left(\tau_{0}\right)}, \\
& \frac{g^{x}(\tau, k)}{e(\tau)} \equiv-i \tilde{G}\left(k ; \tau, \tau_{0}\right) \frac{\delta e\left(\tau_{0}, k\right)}{e\left(\tau_{0}\right)} .
\end{aligned}
$$

The results are presented at two suggested initial times $\tau_{\text {init }}=\{10,20\} / Q_{s}$. We will analyze the response functions at asymptotically small $k$ and in coordinate space in the next two subsections. Analogous results for the response to an initial momentum perturbation are given in the appendix $\mathrm{C}$.

\subsection{The kinetic theory response at asymptotically small $k$}

The most important contribution to the flow arises from the average nuclear geometry, which is smooth on spatial scales of order $c \tau_{\text {init }}$. For this reason the flow due to the average geometry is determined by the $k \rightarrow 0$ limit of the response functions. This section will provide an analytic understanding of this limit, i.e. the intercept of $\tilde{E}\left(k ; \tau, \tau_{0}\right)$ and the slope of $\tilde{G}\left(k ; \tau, \tau_{0}\right)$ in figure 5 . 
First, we will determine how the long wavelength energy perturbations in the transverse plane change as a function of time. Returning to the conservation equations, (3.10a) and (3.10b), and setting $k=0$, we have

$$
\begin{gathered}
\partial_{\tau} e(\tau)=-\frac{e(\tau)+T^{z z}(\tau)}{\tau}, \\
\partial_{\tau} \delta e(\tau)=-\frac{\delta e(\tau)+\delta T^{z z}(\tau)}{\tau} .
\end{gathered}
$$

From these equations the fractional perturbations in the transverse plane $\delta e / e$ remain constant in time in the free streaming limit (where $T^{z z}$ and $\delta T^{z z}$ are zero), and in the hydrodynamic limit (where $T^{z z}$ and $\delta T^{z z}$ are one third $e$ and $\delta e$ ). Outside of these limits $\delta e / e$ is not constant in time.

However, a constant of the motion at $k=0$ can be constructed whenever the hydrodynamic gradient expansion is applicable. Indeed, by dimensional analysis, an all order constitutive equation at $k=0$ must take the following form

$$
T^{z z}=e f\left(e^{1 / 4} \tau\right),
$$

where $f(x)$ is an order one function and $\delta T^{z z}=\partial_{e} T^{z z} \delta e$. Then straightforward steps show that to all orders in the gradient expansion

$$
\lim _{k \rightarrow 0} \frac{\delta e(\tau, k)}{3 e-T^{z z}}=\text { const. }
$$

For conformally invariant theories with $T^{x x}=T^{y y}$ this can be written as

$$
\lim _{k \rightarrow 0} \frac{\delta e(\tau, k)}{e+T^{x x}}=\text { const. }
$$

In figure $6(\mathrm{a})$ we present the time evolution of $\delta e / e$ and $\delta e /\left(e+T^{x x}\right)$ relative to their initial values. For our initial conditions $\delta e /\left(e+T^{x x}\right)$ remains very nearly constant throughout the entire evolution. Using this result, the change in $\delta e / e$ can be determined by the ratio of $\left(e+T^{x x}\right) / e$ at the initial and final times, when $T^{x x}$ is approximately $e / 2$ and $e / 3$ respectively. This reasoning leads to an asymptotic relation between the initial and final energy perturbations

$$
\lim _{\tau \rightarrow \infty} \frac{\delta e(\tau)}{e(\tau)}=\frac{8}{9} \frac{\delta e\left(\tau_{0}\right)}{e\left(\tau_{0}\right)}
$$

which is shown in figure $6(\mathrm{a})$.

Next, we will determine the velocity at (asymptotically) small $k$ as a function of time from the pre-thermal evolution. From the conservation equations for perturbations, (3.10b) and $(3.10 \mathrm{c})$, the momentum perturbations at small $k$ satisfy

$$
\partial_{\tau}\left(\frac{\tau g^{x}}{i k}+\frac{1}{2} \delta e \tau^{2}\right)=-\frac{\tau}{2}\left(2 \delta T^{x x}+\delta T^{z z}-\delta T^{00}\right) .
$$

For conformal theories with $\delta T^{x x}=\delta T^{y y}$ the right hand side of (4.9) is zero and

$$
\frac{\tau g^{x}}{i k}+\frac{1}{2} \delta e \tau^{2}=\text { const. }
$$



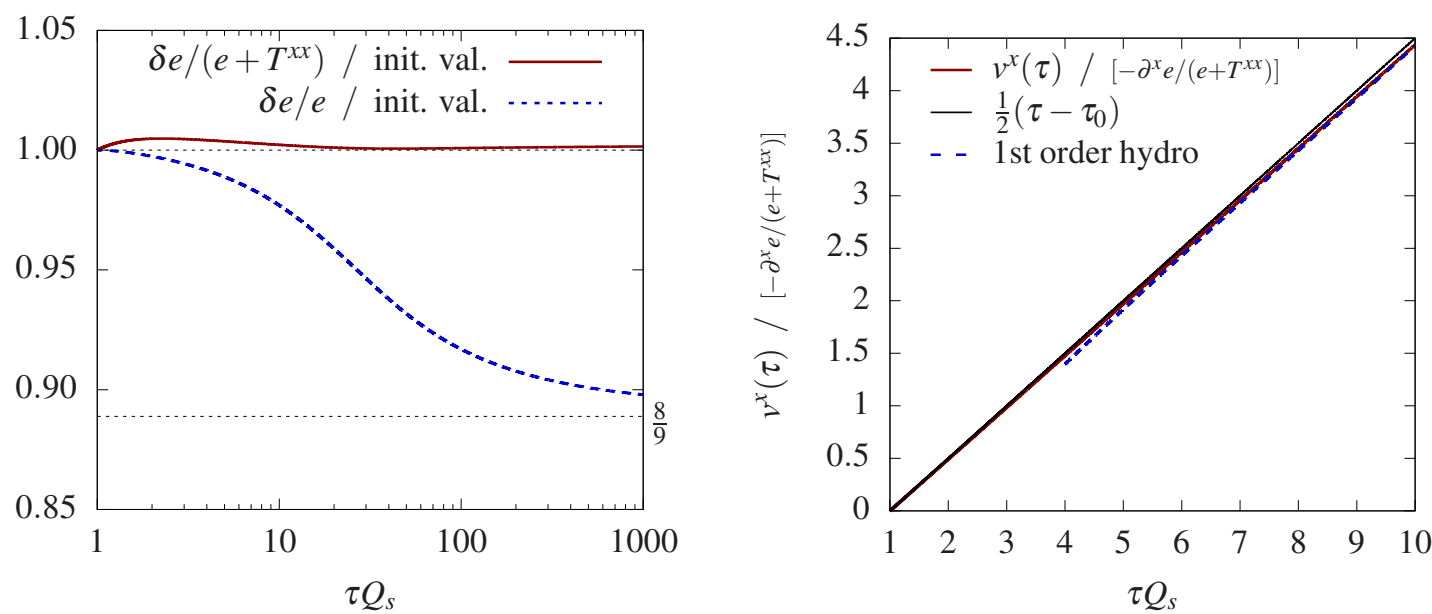

Figure 6. (a) Normalized energy perturbation versus time in the (asymptotically) small $k$ limit. 8/9 is the change in $\left(e+T^{x x}\right) / e$ between free streaming and ideal hydrodynamic limits (see (4.8)). (b) The velocity perturbation versus time in the (asymptotically) small $k$ limit scaled by $-\partial^{x} e /\left(e+T^{x x}\right)$ (see (4.13)). The result is compared to $\frac{1}{2}\left(\tau-\tau_{0}\right)$ (see also ref. [23]) and first order hydrodynamics.

At late times and in coordinate space this condition reads

$$
\frac{T^{0 x}(\tau)}{T^{00}(\tau)}=-\frac{1}{2} \tau \frac{\partial_{x} T^{00}(\tau)}{T^{00}(\tau)},
$$

which was first noted in [23]. Here we have shown that this relation is a consequence of conformal symmetry (see also [23]) and the small $k$ limit.

Using (4.10) and the definition $g^{x}=\left(e+T^{x x}\right) v^{x}$, the velocity as a function of time is given by

$$
\frac{v^{x}}{i k}=-\frac{\tau}{2} \frac{\delta e}{e+T^{x x}}\left(1-\frac{\delta e\left(\tau_{0}\right) \tau_{0}^{2}}{\delta e(\tau) \tau^{2}}\right) .
$$

Thus, after a brief transient period of order $\tau_{0}$, the velocity is directly proportional to time

$$
v^{x}=\frac{\tau}{2}\left(\frac{-\partial^{x} e(\tau, \mathbf{x})}{e(\tau)+T^{x x}(\tau)}\right), \quad \frac{-\partial^{x} e(\tau, \mathbf{x})}{e(\tau)+T^{x x}(\tau)}=\text { const. }
$$

In figure 6(b) we compare the growth of the velocity with time given by (4.12) with a simple estimate based on (4.13). The simple estimate does a remarkably good job for all times.

\subsection{Response in coordinate space}

To construct the initial conditions for hydrodynamics with the correct prethermal evolution, we determine the Green functions $E\left(|\mathbf{x}| ; \tau, \tau_{0}\right)$ and $G\left(|\mathbf{x}| ; \tau, \tau_{0}\right)$ which convert the initial profile of energy perturbations $\delta e\left(\tau_{0}, \mathbf{x}\right)$ to the required energy and momentum fluctuations at thermalization time

$$
\begin{aligned}
& \frac{\delta e(\tau, \mathbf{x})}{e(\tau)}=\int d^{2} \mathbf{x}^{\prime} \frac{\delta e\left(\tau_{0}, \mathbf{x}^{\prime}\right)}{e\left(\tau_{0}\right)} E\left(\left|\mathbf{x}-\mathbf{x}^{\prime}\right| ; \tau, \tau_{0}\right) \\
& \frac{g^{i}(\tau, \mathbf{x})}{e(\tau)}=\int d^{2} \mathbf{x}^{\prime} \frac{\delta e\left(\tau_{0}, \mathbf{x}^{\prime}\right)}{e\left(\tau_{0}\right)} \frac{\left(\mathbf{x}-\mathbf{x}^{\prime}\right)^{i}}{\left|\mathbf{x}-\mathbf{x}^{\prime}\right|} G\left(\left|\mathbf{x}-\mathbf{x}^{\prime}\right| ; \tau, \tau_{0}\right) .
\end{aligned}
$$



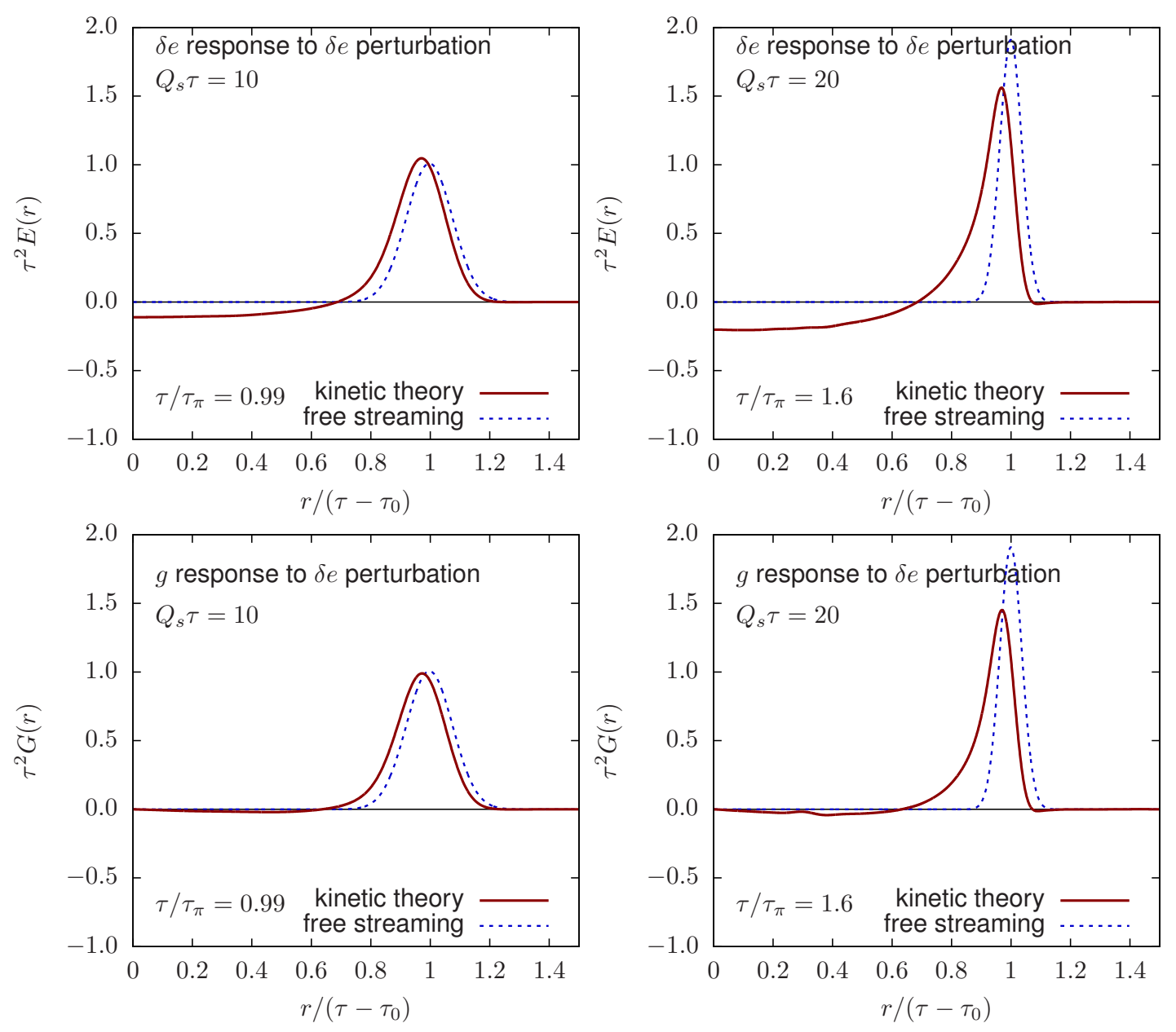

Figure 7. (top) Energy and (bottom) momentum Green functions, (4.14), for initial energy perturbation in coordinate space at (left) $Q_{s} \tau=10$ and (right) $Q_{s} \tau=20$.

Currently hydrodynamic simulations often smooth the initial conditions before starting the hydrodynamic evolution by convolving the energy density with a Gaussian. ${ }^{4}$ (4.14) by contrast smooths the initial conditions in a physical way, and provides an attractive alternative to this ad hoc procedure.

The EKT is applicable for distance scales that are larger than the Compton wavelength of the particles $\sim 1 / Q_{s}$. This limits the accuracy of the Green function in spatial domain that can be reached in a computation based on kinetic theory. In order to fold this uncertainty into our result, we regulate our Green function by convoluting with a Gaussian weight, $e^{-r^{2} / 2 \sigma^{2}} /\left(2 \pi \sigma^{2}\right)$, with $r=|\mathbf{x}|$, and with a width of the order of the initial Compton wavelength $\sigma Q_{s}=0.7$. In momentum space this corresponds to suppressing the large $k$

\footnotetext{
${ }^{4}$ See ref. [14] for a current discussion of the observables that are influenced by this arbitrary regulator.
} 
contributions by an exponential envelope $\exp \left(-\sigma^{2} k^{2} / 2\right)$

$$
\begin{aligned}
& E\left(|\mathbf{x}| ; \tau, \tau_{0}\right)=\int \frac{d^{2} \mathbf{k}}{(2 \pi)^{2}} e^{i \mathbf{k} \cdot \mathbf{x}} e^{-\sigma^{2} k^{2} / 2} E\left(|\mathbf{k}| ; \tau, \tau_{0}\right), \\
& G\left(|\mathbf{x}| ; \tau, \tau_{0}\right)=\int \frac{d^{2} \mathbf{k}}{(2 \pi)^{2}}(-i \hat{k} \cdot \hat{x}) e^{i \mathbf{k} \cdot \mathbf{x}} e^{-\sigma^{2} k^{2} / 2} G\left(|\mathbf{k}| ; \tau, \tau_{0}\right) .
\end{aligned}
$$

The regulated Green functions are shown in figure 7 at the initialization times $\tau_{\text {init }} Q_{s}=$ $\{10,20\}$ (for details of the Fourier transform see appendix B.) At $\tau Q_{s}=10$ the system has spent a significant proportion of the total evolution time with small longitudinal pressure $T^{z z} \approx 0$, and therefore the resulting response is similar to the free streaming prediction (see appendix B). However, the Green function in figure 7(a) is peaked for $r<c\left|\tau-\tau_{0}\right|$, suggesting a slight deflection from the free streaming trajectory. Additionally, the energy perturbation is negative at small $r$, which is indicative of a nascent approach to hydrodynamics. At later times, such as $Q_{s} \tau=20$ in figure $7(\mathrm{~b})$, these differences become more pronounced. Similar features are visible in the momentum response to an initial energy perturbation shown figure $7(\mathrm{c})$ and (d). Finally, in figure 8 we show the Green functions at later times $Q_{s} \tau=50$ and $Q_{s} \tau=500$, and compare to linearized second order hydrodynamics ((3.10) and (3.11)) with initial conditions taken from the $Q_{s} \tau=\{10,20\}$ results. Between $Q_{s} \tau=20$ and $Q_{s} \tau=50$, the hydrodynamics overdamps the high $k$ modes (see also [40]), and the response is broader than the predictions of kinetic theory. However, these Green functions will be convolved with the initial conditions, and thus the resulting hydrodynamic initial state is mostly sensitive to the first moments of these kernels. The moments of the EKT and hydro kernels are determined by the small $k$ behaviour of the response functions, which agree to a few percent (not shown). At later times $Q_{s} \tau=500$, the response is largely determine by Fourier modes in the hydrodynamic regime $k \lesssim 0.1 Q_{s}$, and the EKT and hydro kernels are visually similar.

To summarize, the hydrodynamic evolution sets in early at rather large anisotropies, and the hydrodynamic constitutive equations are approximately satisfied as soon as the $T^{z z}$ starts to significantly deviate from the free streaming expectation, $T^{z z} \approx 0$. For this reason the time interval when the evolution is not described by free streaming or hydrodynamics is comparatively brief (see figure $3(\mathrm{a})$ ), and as hydrodynamics becomes marginally applicable at $Q_{s} \tau_{\text {init }} \sim 10$, the Green function closely resembles the free streaming result. Therefore, an approach where the evolution is described by free streaming until $\tau_{\text {init }}$ seems well motivated $[6,11]$, provided that the correct value of $\tau_{\text {init }}$ is used. However, such an ad hoc approach does not account for some of the qualitative details of the Green function, such as the depletion of the energy density in the interior region.

\section{Discussion}

In this paper, we have provided a bridge between the far-from-equilibrium initial conditions of heavy-ion collisions and hydrodynamized plasma. Our main result is the coordinate space Green functions (see (4.14) and figure 7), which can be used to filter the pre-equilibrium energy density to find the full energy-momentum tensor for hydrodynamics at the initialization time. The procedure can be implemented in complete hydrodynamic simulations, 

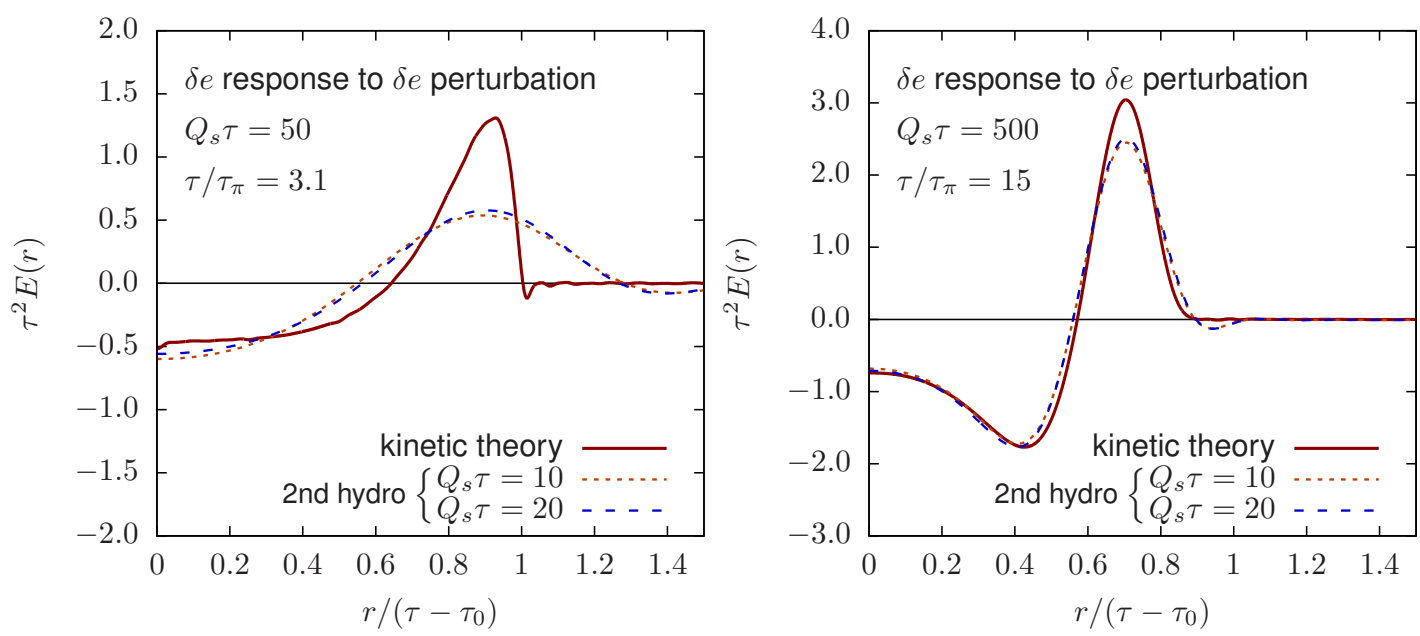

Figure 8. Energy Green functions for initial energy perturbations in coordinate space at late times (a) $Q_{s} \tau=50$ and (b) $Q_{s} \tau=500$. The results are compared to linearized second order hydrodynamics $((3.10)$ and (3.11)), with the initial conditions obtained from kinetic theory at $Q_{s} \tau=10$ and $Q_{s} \tau=20$ (see figure 5).

removing one source of uncertainty. Perhaps more importantly, the approximations in the EKT are compatible with the IP-Glasma setup, and thus the whole evolution from saturated nuclei to hydrodynamics can be comprehensively modelled within a perturbatively controlled framework.

We provide the coordinate space Green functions at two different suggested initialization times, $Q_{s} \tau_{\text {init }}=\{10,20\}$. At the earlier initialization time, $Q_{s} \tau_{\text {init }}=10$, there are significant (though bearable) corrections to the constitutive relations due to nonhydrodynamic modes (see figure 3(a) and figure 4). By $Q_{s} \tau_{\text {init }}=20$ the constitutive relations at small $k$ are well satisfied, and the subsequent evolution is reasonably captured by second order hydrodynamics ${ }^{5}$ (see figure 8 ). The approximate overlap of the two 2 nd order viscous lines in figure 8, which correspond to initializing the hydro at $\tau_{\text {init }} Q_{s}=\{10,20\}$, demonstrates that the subsequent hydrodynamical evolution is indeed rather insensitive to the initialization time. In section 4 we examined the (asymptotically) small $k$ limit of the Green functions, and confirmed (and clarified) a preflow estimate by Vredevoogd and Pratt [23] (see figure 6).

The hydrodynamics that the EKT follows is characterized by the weak coupling value of $\eta / s \approx 0.62$ [25], which is significantly higher than the AdS/CFT result $\eta / s \simeq 0.08$ [41], and current phenomenological estimates, which assume that $\eta / s$ is independent of the temperature. Recent analyses have relaxed the temperature independence of $\eta / s$, and shown that the value of $\eta / s$ at higher temperatures $T \sim 3 T_{c}$ is poorly constrained by data [10]. Since it is the high temperature regime that is most relevant for the transition

\footnotetext{
${ }^{5}$ When examining figure 8, one must remember that the full Green functions will be convolved with the initial conditions, and thus the response of the system is mostly sensitive to the first moments of the kernels in figure 8. The first EKT moments (i.e. the small $k$ behavior of the response) agree with the hydrodynamics to the percent level.
} 
to hydrodynamics, we believe that the current kinetic theory results for the initial stages can be consistent with hydro phenomenology, provided $\eta / s$ decreases towards the strong coupling result as the system cools towards $T_{c}$.

Nevertheless, to apply our results to a hydro simulation with lower viscosity than the perturbative expectation, we note that the two initialization times $\tau_{\text {init }} Q_{s}=\{10,20\}$ correspond in units of the hydro parameters to $\tau_{\text {init }}=\{.99,1.6\} \tau_{\pi}$. The scaled times $\{0.99,1.6\}$ can be used to initialize simulations when the transport coefficients differ. Such an approach is supported by the reasonably good scaling properties of the hydrodynamization times and prethermal evolution as function of the coupling constant when expressed in terms of the hydrodynamic variables [16, 24].

Although our EKT description can be further improved by inclusion of fermionic degrees of freedom and by improving the connection to the early classical evolution, we believe that it already provides a physically sound picture of the approach to hydrodynamics and can be used to initialize all components of the energy-momentum tensor for subsequent hydrodynamic evolution. This eliminates a source of uncertainty in current simulations, and provides a satisfyingly complete description of the early time evolution in heavy ion collisions.

\section{Acknowledgments}

The authors thank François Gelis, Keijo Kajantie, Tuomas Lappi, Risto Paatelainen, JeanFrançois Paquet, and Urs Wiedemann for useful discussions. The simulations were performed using computer resources of UiS and high-performance LIred computing system at the Institute for Advanced Computational Science at Stony Brook University. A.M. and D.T. work was supported in part by the U.S. Department of Energy under Contracts No. DE-FG-88ER40388. Finally, A.M. and D.T. would like to thank CERN Theoretical Physics Department for the hospitality during the short-term visit.

\section{A Collision kernel}

In this appendix we provide additional details on the collision kernels used in (2.3). The collision kernel for the uniform background contains terms arising from elastic $2 \leftrightarrow 2$ scatterings and inelastic $1 \leftrightarrow 2$ collinear splittings

$$
\mathcal{C}[f]=\mathcal{C}_{2 \leftrightarrow 2}[f]+\mathcal{C}_{1 \leftrightarrow 2}[f]
$$

The two collision terms read $[15,16,24]$

$$
\begin{aligned}
\mathcal{C}_{2 \leftrightarrow 2}[f](\mathbf{p})= & \frac{1}{4|\mathbf{p}| \nu_{g}} \int \frac{d^{3} k}{2 k(2 \pi)^{3}} \frac{d^{3} p^{\prime}}{2 p^{\prime}(2 \pi)^{3}} \frac{d^{3} k^{\prime}}{2 k^{\prime}(2 \pi)^{3}} \\
& \times\left|\mathcal{M}\left(\mathbf{p}, \mathbf{k} ; \mathbf{p}^{\prime}, \mathbf{k}^{\prime}\right)\right|^{2}(2 \pi)^{4} \delta^{(4)}\left(P+K-P^{\prime}-K^{\prime}\right) \\
& \times\left\{f_{\mathbf{p}} f_{\mathbf{k}}\left[1+f_{\mathbf{p}^{\prime}}\right]\left[1+f_{\mathbf{k}^{\prime}}\right]-f_{\mathbf{p}^{\prime}} f_{\mathbf{k}^{\prime}}\left[1+f_{\mathbf{p}}\right]\left[1+f_{\mathbf{k}}\right]\right\}
\end{aligned}
$$


and

$$
\begin{aligned}
\mathcal{C}^{1 \leftrightarrow 2}[f](\mathbf{p})= & \frac{(2 \pi)^{3}}{2|\mathbf{p}|^{2} \nu_{g}} \int_{0}^{\infty} d p^{\prime} d k^{\prime} \delta\left(|\mathbf{p}|-p^{\prime}-k^{\prime}\right) \gamma\left(\mathbf{p} ; p^{\prime} \hat{\mathbf{p}}, k^{\prime} \hat{\mathbf{p}}\right) \\
& \times\left\{f_{\mathbf{p}}\left[1+f_{p^{\prime} \hat{\mathbf{p}}}\right]\left[1+f_{k^{\prime} \hat{\mathbf{p}}}\right]-f_{p^{\prime} \hat{\mathbf{p}}} f_{k^{\prime}}\left[1+f_{\mathbf{p}}\right]\right\} \\
+ & \frac{(2 \pi)^{3}}{|\mathbf{p}|^{2} \nu_{g}} \int_{0}^{\infty} d p^{\prime} d k \delta\left(|\mathbf{p}|+k-p^{\prime}\right) \gamma\left(p^{\prime} \hat{\mathbf{p}} ; \mathbf{p}, k \hat{\mathbf{p}}\right) \\
& \times\left\{f_{\mathbf{p}} f_{k \hat{\mathbf{p}}}\left[1+f_{p^{\prime} \hat{\mathbf{p}}}\right]-f_{p^{\prime} \hat{\mathbf{p}}}\left[1+f_{\mathbf{p}}\right]\left[1+f_{k \hat{\mathbf{p}}}\right]\right\}
\end{aligned}
$$

where $\hat{\mathbf{p}}$ is the unit vector parallel to $\mathbf{p}$, and capital letters denote null 4 -vectors, i.e. $P^{0} \equiv|\mathbf{p}|$. The effective elastic $|\mathcal{M}|^{2}$ and inelastic $\gamma$ scattering matrix elements contain non-trivial structures arising from the soft and collinear divergences, which are dynamically regulated by the in-medium physics.

For the most of kinematics the effective elastic scattering element is given by ${ }^{6}$

$$
|\mathcal{M}|^{2}=2 \lambda^{2} \nu_{g}\left(9+\frac{(s-t)^{2}}{u^{2}}+\frac{(u-s)^{2}}{t^{2}}+\frac{(t-u)^{2}}{s^{2}}\right) .
$$

For a soft gluon exchange with the momentum transfer $q=\left|\mathbf{p}^{\prime}-\mathbf{p}\right|$ in $t$-channel (or $q=\left|\mathbf{p}^{\prime}-\mathbf{k}\right|$ in $u$-channel) the collision matrix is proportional to $\propto 1 /\left(q^{2}\right)^{2}$, and thus suffers from a soft Coulomb divergence. It is regulated by replacing

$$
q^{2} t \rightarrow\left(q^{2}+2 \xi_{0}^{2} m^{2}\right) t
$$

in the denominators of divergent terms (similarly for the $u$-channel). Here $m^{2}$ is the thermal asymptotic mass of the gluon defined as

$$
m^{2}=2 \lambda \int \frac{d^{3} p}{(2 \pi)^{3}} \frac{f_{\mathbf{p}}}{|\mathbf{p}|}
$$

The coefficient $\xi_{0}=e^{5 / 6} / \sqrt{8}$ is fixed so that the matrix element reproduces the drag and momentum diffusion properties of soft scattering at leading order for isotropic distributions $f_{\mathbf{p}}[38]$.

The effective splitting kernel reads

$$
\gamma\left(p \hat{\mathbf{p}} ; p^{\prime} \hat{\mathbf{p}}, k^{\prime} \hat{\mathbf{p}}\right)=\frac{p^{4}+p^{\prime 4}+k^{\prime 4}}{p^{3} p^{\prime 3} k^{\prime 3}} \frac{\nu_{g} \lambda}{8(2 \pi)^{4}} \int \frac{d^{2} h}{(2 \pi)^{2}} 2 \mathbf{h} \cdot \operatorname{Re} \mathbf{F}
$$

where the equation for $\mathbf{F}$ accounts for splitting due to multiple scatterings with transverse momentum exchange $q$, and momentum non-collinearity

$$
\begin{aligned}
2 \mathbf{h}= & i \delta E(\mathbf{h}) \mathbf{F}(\mathbf{h})+\frac{\lambda T_{*}}{2} \int \frac{d^{2} q_{\perp}}{(2 \pi)^{2}} \mathcal{A}\left(\mathbf{q}_{\perp}\right) \\
& \times\left[3 \mathbf{F}(\mathbf{h})-\mathbf{F}\left(\mathbf{h}-p^{\prime} \mathbf{q}_{\perp}\right)-\mathbf{F}\left(\mathbf{h}-k^{\prime} \mathbf{q}_{\perp}\right)-\mathbf{F}\left(\mathbf{h}+p \mathbf{q}_{\perp}\right)\right] .
\end{aligned}
$$

\footnotetext{
${ }^{6}$ Equations (A.4) and (A.8) have some minor typos corrected compared to refs. [16, 24].
} 
with $T_{*}=\frac{\lambda}{m^{2}} \int \frac{d^{3} p}{(2 \pi)^{3}} f_{\mathbf{p}}\left(1+f_{\mathbf{p}}\right)$, and $\delta E=m^{2} / 2 p^{\prime}+m^{2} / 2 k^{\prime}-m^{2} / 2 p+\mathbf{h}^{2} / 2 p k^{\prime} p^{\prime}$. In the isotropic screening approximation

$$
\mathcal{A}\left(\mathbf{q}_{\perp}\right)=\left(\frac{1}{\mathbf{q}_{\perp}^{2}}-\frac{1}{\mathbf{q}_{\perp}^{2}+2 m^{2}}\right) .
$$

Both $m^{2}$ and $T_{*}$ are self-consistently evaluated at each time step.

The linearized collision kernels are obtained trivially by replacing $f \rightarrow \bar{f}+\delta f$ in the integrands of (A.2) and (A.3) and linearizing in $\delta f$. In addition one has to take into account the linear variation of the thermal mass $\delta m^{2}$ and the effective temperature $\delta T_{*}$ in the scattering matrix elements (A.4) and (A.7)

$$
\begin{aligned}
\delta m^{2} & =2 \lambda \int \frac{d^{3} p}{(2 \pi)^{3}} \frac{\delta f_{\mathbf{p}}}{|\mathbf{p}|}, \\
\delta T_{*} & =\frac{\lambda}{m^{2}} \int \frac{d^{3} p}{(2 \pi)^{3}} \delta f_{\mathbf{p}}\left(1+2 f_{\mathbf{p}}\right)-\frac{\delta m^{2}}{m^{2}} T_{*} .
\end{aligned}
$$

where $m^{2}$ and $T_{*}$ are evaluated from the unperturbed background distribution.

\section{B Fourier transform of Green functions}

Here we provide details of performing Fourier transforms in (4.15) and (4.16) to obtain spatial Green functions shown in figures 7 and 8. The two dimensional Fourier transforms can be straightforwardly reduced to one dimensional Hankel transforms

$$
\begin{aligned}
& E\left(|\mathbf{x}| ; \tau, \tau_{0}\right)=\int_{0}^{\infty} \frac{d k}{2 \pi} k \tilde{E}\left(k ; \tau, \tau_{0}\right) e^{-\sigma^{2} k^{2} / 2} J_{0}(k|\mathbf{x}|) \\
& G\left(|\mathbf{x}| ; \tau, \tau_{0}\right)=\int_{0}^{\infty} \frac{d k}{2 \pi} k \tilde{G}\left(k ; \tau, \tau_{0}\right) e^{-\sigma^{2} k^{2} / 2} J_{1}(k|\mathbf{x}|)
\end{aligned}
$$

Integrals in (B.1) and (B.2) were done numerically by using cubic interpolation for $\tilde{E}$ and $\tilde{G}$ within the available range of wavenumbers $k \in[0,4] Q_{s}$ To avoid the oscillatory behaviour due to a sharp $k$ cut-off at $k=4 Q_{s}$, we extrapolated the Green functions until the Gaussian envelope $e^{-\sigma^{2} k^{2} / 2}$ smoothly cuts off the integral. For extrapolation at large $k$ we used functional forms motivated by free streaming results: $C_{0} J_{0}\left(v_{0}|k|\left(\tau-\tau_{0}\right)\right)$ and $C_{1} J_{1}\left(v_{1}|k|\left(\tau-\tau_{0}\right)\right)$, where coefficients $C_{i}$ and $v_{i}$ were fitted to match the oscillatory behaviour of Green functions at the largest available $k$. For $Q_{s} \tau=\{10,20,50\}$ we used $Q_{s} \sigma=0.7$ for the envelope corresponding roughly to the smallest scales the EKT can resolve. For $Q_{s} \tau=500$, perturbations with large wavenumbers were sufficiently suppressed by EKT evolution that no extrapolation was necessary.

For early times and large values of $k$ the collision terms in the Boltzmann equation (2.3) can be neglected and the system is freely streaming. For particle distributions that are highly anisotropic in $z$ direction $\left(\mathcal{P}_{L} \ll \mathcal{P}_{T}\right)$, but isotropic in $x y$-plane, energy perturbations are propagating in circular wavefronts at the velocity $v$ of constituent particles (for massless gluons $v=c$ ). In such free streaming evolution energy perturbations at time $\tau$ and position 

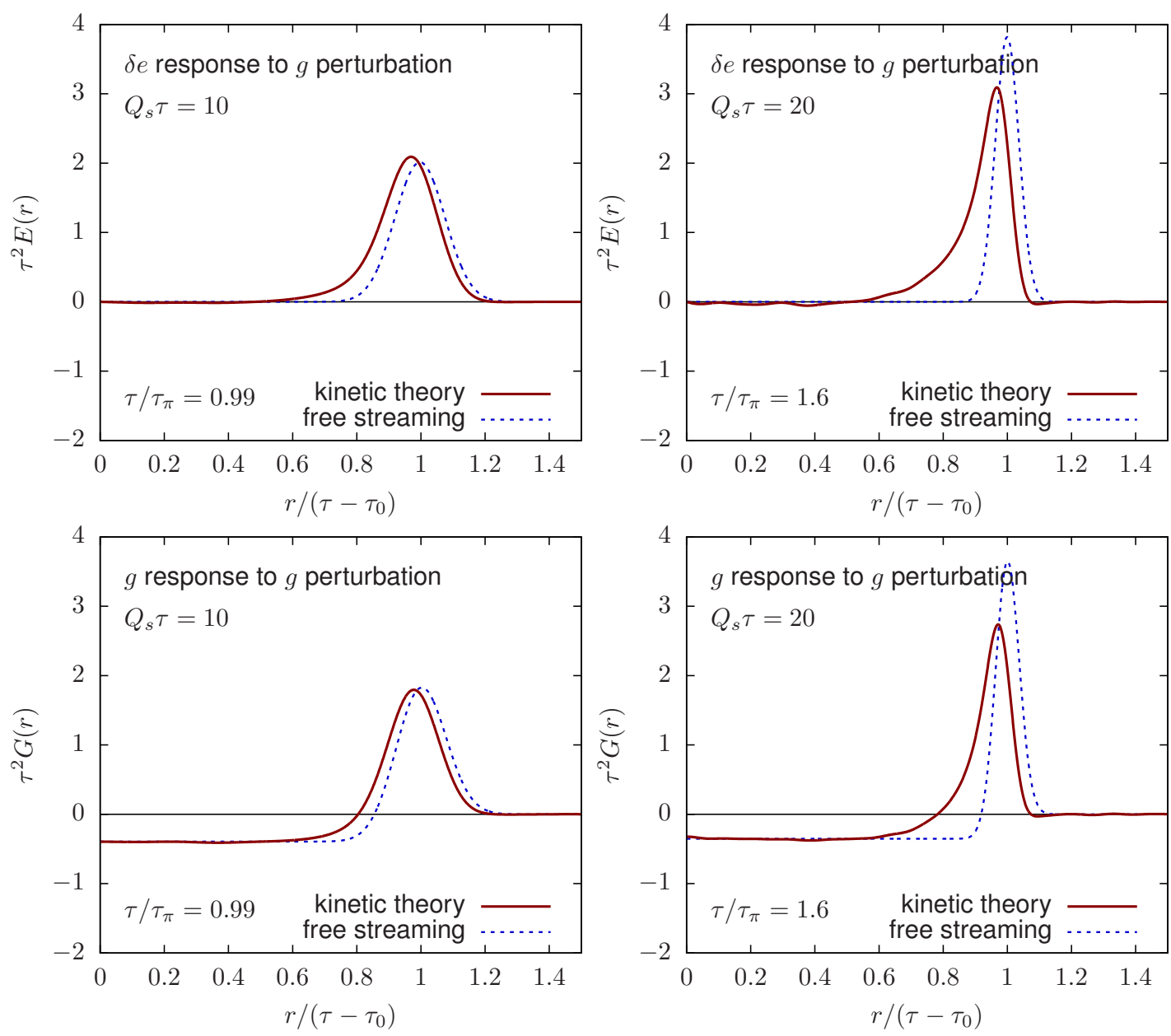

Figure 9. (top) Energy and (bottom) momentum Green functions, (C.3), for initial momentum perturbation in coordinate space at (left) $Q_{s} \tau=10$ and (right) $Q_{s} \tau=20$.

$\mathbf{x}$ are equal to the average of energy perturbations at $\tau_{0}$ on a circle $\left|\mathbf{x}-\mathbf{x}^{\prime}\right|=c\left|\tau-\tau_{0}\right|[6]$. Thus, free streaming Green functions in coordinate space are

$$
E\left(|\mathbf{x}| ; \tau, \tau_{0}\right)=G\left(|\mathbf{x}| ; \tau, \tau_{0}\right)=\frac{1}{2 \pi|\mathbf{x}|} \delta\left(\left|\tau-\tau_{0}\right|-|\mathbf{x}|\right) .
$$

Here we also quote the free streaming result for the response to initial momentum perturbations defined in appendix $\mathrm{C}$

$$
\begin{aligned}
& E\left(|\mathbf{x}|, \tau, \tau_{0}\right)=\frac{2}{2 \pi|\mathbf{x}|} \delta\left(\left|\tau-\tau_{0}\right|-|\mathbf{x}|\right) \\
& G\left(|\mathbf{x}|, \tau, \tau_{0}\right)=\frac{2}{2 \pi|\mathbf{x}|} \delta\left(\left|\tau-\tau_{0}\right|-|\mathbf{x}|\right)-\frac{2}{2 \pi} \frac{1}{|\mathbf{x}|} \theta\left(\left|\tau-\tau_{0}\right|-|\mathbf{x}|\right)
\end{aligned}
$$

Free streaming Green functions shown in figure 7 and figure 9 were also folded in with a Gaussian regulator as discussed above. 


\section{Initial velocity perturbations}

In this appendix we summarize results for the EKT response to initial momentum perturbation, (3.7). Mirroring the discussion in section 4, we define Green functions for initial momentum perturbations in $k$-space as follows

$$
\begin{aligned}
& \frac{\delta e(\tau, k)}{e(\tau)}=-i \tilde{E}\left(k ; \tau, \tau_{0}\right) \frac{g^{x}\left(\tau_{0}, k\right)}{e\left(\tau_{0}\right)}, \\
& \frac{g^{x}(\tau, k)}{e(\tau)}=\tilde{G}\left(k ; \tau, \tau_{0}\right) \frac{g^{x}\left(\tau_{0}, k\right)}{e\left(\tau_{0}\right)} .
\end{aligned}
$$

By performing Fourier transform and regularization (see appendix B for details) we obtain Green functions in coordinate space, which can be used to propagate initial momentum fluctuations to energy and momentum perturbations at a given thermalization time

$$
\begin{aligned}
& \frac{\delta e(\tau, \mathbf{x})}{e(\tau)}=\int d^{2} \mathbf{x}^{\prime} \frac{\left(\mathbf{x}-\mathbf{x}^{\prime}\right)^{i}}{\left|\mathbf{x}-\mathbf{x}^{\prime}\right|} \frac{g^{i}\left(\tau_{0}, \mathbf{x}^{\prime}\right)}{e\left(\tau_{0}\right)} E\left(\left|\mathbf{x}-\mathbf{x}^{\prime}\right| ; \tau, \tau_{0}\right), \\
& \frac{g^{i}(\tau, \mathbf{x})}{e(\tau)}=\int d^{2} \mathbf{x}^{\prime} \frac{g^{i}\left(\tau_{0}, \mathbf{x}^{\prime}\right)}{e\left(\tau_{0}\right)} G\left(\left|\mathbf{x}-\mathbf{x}^{\prime}\right| ; \tau, \tau_{0}\right) .
\end{aligned}
$$

The regulated Green functions for initial momentum perturbations are shown in figure 9 . As a comparison we also include Green functions for free streaming evolution, (B.4) and (B.5).

Energy and momentum Green functions shown in figure 7 and figure 9 make a complete set of response functions necessary to propagate arbitrary initial energy and momentum perturbations from the far-from-equilibrium initial conditions to times $Q_{s} \tau=\{10,20\}$ when hydrodynamics becomes applicable. Crucially, at these times one can use hydrodynamic constitutive equations (3.11) to initialize all components of the energy momentum tensor.

Open Access. This article is distributed under the terms of the Creative Commons Attribution License (CC-BY 4.0), which permits any use, distribution and reproduction in any medium, provided the original author(s) and source are credited.

\section{References}

[1] U. Heinz and R. Snellings, Collective flow and viscosity in relativistic heavy-ion collisions, Ann. Rev. Nucl. Part. Sci. 63 (2013) 123 [arXiv:1301.2826] [InSPIRE].

[2] M. Luzum and H. Petersen, Initial state fluctuations and final state correlations in relativistic heavy-ion collisions, J. Phys. G 41 (2014) 063102 [arXiv:1312.5503] [InSPIRE].

[3] D.A. Teaney, Viscous hydrodynamics and the quark gluon plasma, arXiv:0905.2433 [INSPIRE].

[4] R. Baier, P. Romatschke, D.T. Son, A.O. Starinets and M.A. Stephanov, Relativistic viscous hydrodynamics, conformal invariance and holography, JHEP 04 (2008) 100 [arXiv:0712.2451] [INSPIRE].

[5] J.E. Bernhard, P.W. Marcy, C.E. Coleman-Smith, S. Huzurbazar, R.L. Wolpert and S.A. Bass, Quantifying properties of hot and dense QCD matter through systematic model-to-data comparison, Phys. Rev. C 91 (2015) 054910 [arXiv:1502.00339] [INSPIRE]. 
[6] J. Liu, C. Shen and U. Heinz, Pre-equilibrium evolution effects on heavy-ion collision observables, Phys. Rev. C 91 (2015) 064906 [Erratum ibid. C 92 (2015) 049904] [arXiv: 1504.02160] [INSPIRE].

[7] W. van der Schee, P. Romatschke and S. Pratt, Fully dynamical simulation of central nuclear collisions, Phys. Rev. Lett. 111 (2013) 222302 [arXiv: 1307.2539] [INSPIRE].

[8] P. Romatschke, Light-heavy ion collisions: a window into pre-equilibrium QCD dynamics?, Eur. Phys. J. C 75 (2015) 305 [arXiv: 1502.04745] [InSPIRE].

[9] A. Kurkela, Initial state of heavy-ion collisions: isotropization and thermalization, in $25^{\text {th }}$ International Conference on Ultra-Relativistic Nucleus-Nucleus Collisions (Quark Matter 2015), Kobe Japan September 27-October 32015 [arXiv:1601.03283] [INSPIRE].

[10] H. Niemi, K.J. Eskola and R. Paatelainen, Event-by-event fluctuations in a perturbative QCD + saturation + hydrodynamics model: determining QCD matter shear viscosity in ultrarelativistic heavy-ion collisions, Phys. Rev. C 93 (2016) 024907 [arXiv: 1505.02677] [INSPIRE].

[11] W. Broniowski, W. Florkowski, M. Chojnacki and A. Kisiel, Free-streaming approximation in early dynamics of relativistic heavy-ion collisions, Phys. Rev. C 80 (2009) 034902 [arXiv: 0812.3393] [INSPIRE].

[12] B. Schenke, P. Tribedy and R. Venugopalan, Fluctuating glasma initial conditions and flow in heavy ion collisions, Phys. Rev. Lett. 108 (2012) 252301 [arXiv:1202.6646] [INSPIRE].

[13] H. Niemi and G.S. Denicol, How large is the Knudsen number reached in fluid dynamical simulations of ultrarelativistic heavy ion collisions?, arXiv:1404.7327 [INSPIRE].

[14] J. Noronha-Hostler, J. Noronha and M. Gyulassy, Sensitivity of flow harmonics to subnucleon scale fluctuations in heavy ion collisions, Phys. Rev. C 93 (2016) 024909 [arXiv: 1508.02455] [INSPIRE].

[15] P.B. Arnold, G.D. Moore and L.G. Yaffe, Effective kinetic theory for high temperature gauge theories, JHEP 01 (2003) 030 [hep-ph/0209353] [INSPIRE].

[16] A. Kurkela and Y. Zhu, Isotropization and hydrodynamization in weakly coupled heavy-ion collisions, Phys. Rev. Lett. 115 (2015) 182301 [arXiv:1506.06647] [INSPIRE].

[17] E. Iancu, A. Leonidov and L. McLerran, The color glass condensate: an introduction, in QCD perspectives on hot and dense matter. Proceedings, NATO Advanced Study Institute, Summer School, Cargese France August 6-18 2001, pg. 73 [hep-ph/0202270] [INSPIRE].

[18] E. Iancu and R. Venugopalan, The color glass condensate and high-energy scattering in QCD, in Quark gluon plasma, R.C. Hwa et al. eds., (2003), pg. 249 [hep-ph/0303204] [INSPIRE].

[19] F. Gelis, E. Iancu, J. Jalilian-Marian and R. Venugopalan, The color glass condensate, Ann. Rev. Nucl. Part. Sci. 60 (2010) 463 [arXiv:1002.0333] [InSPIRE].

[20] F. Gelis, T. Lappi and R. Venugopalan, High energy scattering in quantum chromodynamics, Int. J. Mod. Phys. E 16 (2007) 2595 [arXiv:0708.0047] [InSPIRE].

[21] T. Lappi, Gluon spectrum in the glasma from JIMWLK evolution, Phys. Lett. B 703 (2011) 325 [arXiv: 1105.5511] [INSPIRE].

[22] A. Mazeliauskas and D. Teaney, Subleading harmonic flows in hydrodynamic simulations of heavy ion collisions, Phys. Rev. C 91 (2015) 044902 [arXiv:1501.03138] [INSPIRE].

[23] J. Vredevoogd and S. Pratt, Universal flow in the first stage of relativistic heavy ion collisions, Phys. Rev. C 79 (2009) 044915 [arXiv:0810.4325] [InSPIRE]. 
[24] L. Keegan, A. Kurkela, P. Romatschke, W. van der Schee and Y. Zhu, Weak and strong coupling equilibration in non-Abelian gauge theories, JHEP 04 (2016) 031 [arXiv: 1512.05347] [INSPIRE].

[25] P.B. Arnold, G.D. Moore and L.G. Yaffe, Transport coefficients in high temperature gauge theories. 2. Beyond leading log, JHEP 05 (2003) 051 [hep-ph/0302165] [INSPIRE].

[26] M.A. York and G.D. Moore, Second order hydrodynamic coefficients from kinetic theory, Phys. Rev. D 79 (2009) 054011 [arXiv:0811.0729] [INSPIRE].

[27] A. Kovner, L.D. McLerran and H. Weigert, Gluon production from non-Abelian Weizsacker-Williams fields in nucleus-nucleus collisions, Phys. Rev. D 52 (1995) 6231 [hep-ph/9502289] [INSPIRE].

[28] T. Lappi and L. McLerran, Some features of the glasma, Nucl. Phys. A 772 (2006) 200 [hep-ph/0602189] [INSPIRE].

[29] T. Epelbaum and F. Gelis, Pressure isotropization in high energy heavy ion collisions, Phys. Rev. Lett. 111 (2013) 232301 [arXiv:1307.2214] [INSPIRE].

[30] G. Chen, R.J. Fries, J.I. Kapusta and Y. Li, Early time dynamics of gluon fields in high energy nuclear collisions, Phys. Rev. C 92 (2015) 064912 [arXiv:1507.03524] [INSPIRE].

[31] M. Li and J.I. Kapusta, Pressure anisotropy in heavy ion collisions from color glass condensate, arXiv: 1602.09060 [INSPIRE].

[32] A. Kurkela and G.D. Moore, Thermalization in weakly coupled non-Abelian plasmas, JHEP 12 (2011) 044 [arXiv: 1107.5050] [INSPIRE].

[33] A. Kurkela and G.D. Moore, Bjorken flow, plasma instabilities and thermalization, JHEP 11 (2011) 120 [arXiv:1108.4684] [INSPIRE].

[34] J. Berges, K. Boguslavski, S. Schlichting and R. Venugopalan, Universal attractor in a highly occupied non-Abelian plasma, Phys. Rev. D 89 (2014) 114007 [arXiv:1311.3005] [INSPIRE].

[35] R. Baier, A.H. Mueller, D. Schiff and D.T. Son, 'Bottom up' thermalization in heavy ion collisions, Phys. Lett. B 502 (2001) 51 [hep-ph/0009237] [INSPIRE].

[36] A.H. Mueller and D.T. Son, On the equivalence between the Boltzmann equation and classical field theory at large occupation numbers, Phys. Lett. B 582 (2004) 279 [hep-ph/0212198] [INSPIRE].

[37] S. Jeon, The Boltzmann equation in classical and quantum field theory, Phys. Rev. C 72 (2005) 014907 [hep-ph/0412121] [INSPIRE].

[38] M.C. Abraao York, A. Kurkela, E. Lu and G.D. Moore, UV cascade in classical Yang-Mills theory via kinetic theory, Phys. Rev. D 89 (2014) 074036 [arXiv:1401.3751] [INSPIRE].

[39] M. Luzum and P. Romatschke, Conformal relativistic viscous hydrodynamics: applications to RHIC results at $\sqrt{s_{N N}}=200 \mathrm{GeV}$, Phys. Rev. C 78 (2008) 034915 [Erratum ibid. $\mathbf{C} \mathbf{7 9}$ (2009) 039903] [arXiv:0804.4015] [INSPIRE].

[40] P. Romatschke, Retarded correlators in kinetic theory: branch cuts, poles and hydrodynamic onset transitions, Eur. Phys. J. C 76 (2016) 352 [arXiv:1512.02641] [INSPIRE].

[41] G. Policastro, D.T. Son and A.O. Starinets, The shear viscosity of strongly coupled $N=4$ supersymmetric Yang-Mills plasma, Phys. Rev. Lett. 87 (2001) 081601 [hep-th/0104066] [INSPIRE]. 